\title{
Effective Interactions in the Even Configurations of the Third Spectra of the Iron Group*
}

\author{
Y. Shadmi, ** E. Caspi, *** and J. Oreg***
}

(November 19 1968)

\begin{abstract}
Four hundred eighty-three levels belonging to the configurations $3 d^{n}+3 d^{n-1} 4 s$ of all third spectra of the iron group were calculated, and 334 observed levels were fitted to them. In addition to the usually used approximation, we first introduced a complete set of two-body and three-body effective-interaction parameters between $3 d$ electrons. Using only two-body effective interactions, we obtained a rms error of $175 \mathrm{~cm}^{-1}$; while the addition of three-body effective interactions reduced the rms error to $46 \mathrm{~cm}^{-1}$. When a parameter representing three-body effective interaction between $3 d$ and $4 s$ electrons was also introduced, the rms error was reduced to $38 \mathrm{~cm}^{-1}$
\end{abstract}

Key words: Atomic theory; iron group; third spectra.

\section{Introduction}

In the present paper, we describe a systematic treatment of the even configurations in all third spectra of the iron group. Section 2 of the present paper is completely analogous to the treatment already performed for the second spectra [1]. ${ }^{1}$ In that treatment, a considerable improvement in our theoretical approximation was achieved by including a set of parameters which completely represent all possible effective-interactions between $3 d$ electrons.

The first author of the present paper published a series of papers in which he reported on systematic theoretical investigations of various sequences of spectra of transition elements [2-5]. In all these papers, practically the same approximation was used: The Slater approximation was improved by including the interaction between the three configurations $3 d^{n}, 3 d^{n-1} 4 s, 3 d^{n-2} 4 s^{2}$; the $\alpha \mathrm{L}(\mathrm{L}+1)$ correction; and the spin-orbit interaction.

The result of a long theoretical development to which many physicists (e.g., Bacher and Goudsmit [6], Trees [7-9], Racah [10-11], Rajnak and Wybourne [12], Stein [13], Judd [14], and Feneuille [15]) made their contributions, was that in order to completely describe the second-order perturbations of a $d^{n}$ con-

\footnotetext{
*An invited paper. This paper was supported in part by the National Bureau of Standards. Washington, D.C. 20234

**Present address: Goddard Space Flight Center, National Aeronautics and Space Administration, Greenbelt, Maryland 20770. (On sabbatical leave from The Hebrew University of Jerusalem, Israel.)

***The Hebrew University of Jerusalem, Israel.

1 Figures in brackets refer to the literature references at the end of this paper.

${ }^{2}$ In all the references cited here, the expressions "mean error" and "mean deviation" were used for the presentiy defined "rms error" and "rms deviation," respectively.
}

figuration by configurations lying far from it, one needs to improve the Slater approximation. by adding two parameters of two-body effective-interactions (namely $\alpha$ and $\beta$ ), and two parameters of three-body interactions, $T$ and $T x$.

A more detailed description of the above mentioned development, and a detailed definition of the new above mentioned parameters, as well as of the old ones, are given in reference [1]. For that reason, in this paper, we shall only repeat briefly that the two two-body effective-interaction parameters $\alpha$ anb $\beta$ are defined as follows: $\alpha$ is the parameter multiplying the $\mathrm{L}(\mathrm{L}+\mathrm{l})$ correction; $\beta$ is the coefficient of the $Q$-correction (where $Q$ is the seniority operator [16]). For the two three-body effective-interaction parameters, we shall only mention that $T$ is the parameter which fully represents the perturbation of $3 s^{2} 3 d^{n}$ by $3 s 3 d^{n+1}$, while $T x$ is an additional independent three-body-interaction parameter which is necessary in order to make the set of parameters complete. Its formal definition is also given in reference [1]. $P x$ is the parameter representing the three-body effective interaction between $3 d$ and $4 s$ electrons. Its full definition is given in section 3 . We would also like to emphasize that we measured the accuracy of the fit between the theoretical and experimental levels by the rms error ${ }^{2} \Delta$ which is defined by the equation:

$$
\Delta=\left[\left(\sum_{i} \delta_{i}^{2}\right) /(n-m)\right]^{1 / 2}
$$

where $\delta_{i}$ is a single deviation; $n$ is the number of observed data; and $m$ is the number of free parameters 
used in the calculation. Contrary to the rms deviation ${ }^{2}$ $\bar{\delta}$ which is defined by the equation

$$
\bar{\delta}=\left[\left(\sum_{i} \delta_{i}^{2}\right) / n\right]^{1 / 2},
$$

the rms error takes into account the effect of the addition of more free parameters. Thus, if the addition of a new parameter causes an essential decrease of the rms error, it means that this parameter really has physical significance. For a more detailed description of the above mentioned notations, definitions and considerations, the reader is referred to reference [1].

In sections 2 and 3 of this paper, the introduction of all the above mentioned effective-interactions is described. In section 4 , we investigate the effects of also adding the effective-interaction between $3 d$ and $4 s$ electrons. The results achieved in this paper are summarized and discussed in section 5 .

The main purposes of the calculations to be described in the following sections are:

(a) To check if the addition of the new effectiveinteractions actually improves our approximation (i.e., reduces the rms error); and if it does, to find the numerical values of the new parameters and to evaluate a new optimal set of numerical values for all the interaction parameters.

(b) To study the behavior of the parameters in different spectra and different configurations, and to evaluate the relative importance of the various effective-interaction parameters.

(c) To use the improved approximation for a more accurate prediction of the unobserved levels and in some cases, also, for a better critical examination of some experimental levels.

\section{The Use of a Complete Set of Effective- Interactions Between 3d Electrons}

The starting point for our present calculation was the final results of the first author's above mentioned paper [3] on the third spectra of the iron group. In that paper, the Slater approximation was improved by the addition of the $\alpha \mathrm{L}(\mathrm{L}+1)$ correction and the spin-orbit interaction. In this case, for each spectrum only two configurations, $3 d^{n}$ and $3 d^{n-1} 4 s$, were included in the calculation. The third configuration, $3 d^{n-2} 4 s^{2}$, is very high and experimentally unknown. It is certain that its interaction with the two lower configurations is negligible. In fact, in that previous calculation, we didn't succeed in including the interaction between the two lower configurations, since its effect is apparently so small that in the least-squares calculation, it didn't determine the configuration interaction parameter $H$.

In the previous work [3], 483 levels were calculated and fitted to 322 observed levels with the use of 30 free parameters, and the rms error was $160 \mathrm{~cm}^{-1}$.

The main source of the experimental material used in the previous work [3], as well as in our present paper, is Moore's compilation [17]. Additional sources not used in the previous work are explicitly referred to in appropriate paragraphs of our present paper.

It should be noted that in that calculation [3], the observed level $d^{6} a^{1} \mathrm{~S}$ of Fe III was excluded from the least-squares fit because of a very large deviation. Had it been included in the least-squares fit, the rms error achieved with the previous approximation would have been $184 \mathrm{~cm}^{-1}$. (For the detailed history of this $a^{1} \mathrm{~S}$ see reference [18].)

We also have to mention that in the previous work [3], the calculation was concluded by expressing all the interaction parameters by simple interpolation formulas (usually linear functions of the atomic number, and in some cases, with a small quadratic correction). In this way, all the spectra of that sequence were simultaneously treated in one total least-squares calculation in which the coefficients of the interpolation formulas took the role of free parameters. For any interaction parameter $P$, such a formula was of the form

where

$$
P=P(n)=P_{0}+P_{1} \cdot x+P_{2} \cdot y
$$

$$
\begin{aligned}
& x=n-6 \\
& y=x^{2}-10
\end{aligned}
$$

and $n$ is the total number of $3 d$ and $4 s$ electrons for each spectrum. For most of the parameters, we could put $P_{2}=0$. In the present calculation, we used the interpolative method from the beginning.

As initial values for the general parameters, we took the final values of the previous work [3] with the following changes: for the parameter $T$ (which was not used in the previous work), we took a value which was the average between the value obtained by us in reference [1] and the one obtained by Trees in his paper on Fe III [8]. For $\beta$, we took the values obtained in reference [1]; and according to our experience with the second spectra of the iron group, we took an initial value for $\alpha$ only one-half of the value it assumed in the previous work [3]. For the same reason, we increased the value for the parameter $C$ by about 10 percent. For the new parameter $T x$, we assigned the initial value zero.

In principle, we allowed a maximum number of 44 free parameters, including 10 additive parameters, one for each spectrum, and 34 coefficients of the interpolation formulas for the interaction parameters. We call these coefficients "general parameters." It should be noted that in the previous work [3], we had only 30 free parameters. Later, we shall see that in the present work, too, the final number of meaningful parameters is much smaller than the maximum number of 44 just mentioned. The values assumed by the "general parameters" in our various L.S. calculations are given in table 1 . We did not report the values of the 10 additive parameters.

The whole set of initial parameters for our first diagonalization is given in table 1 in the column headed Diag. I. The derivatives of Diag. I were used for three least-squares calculations designated by L.S. la, L.S. 1b and L.S. 1c. In all of these calculations, the observed levels fitted to the calculated ones are the 
TABLE 1. Parameters of the various stages of the calculation

(parameter values are given in units of $\mathrm{cm}^{-1}$ )

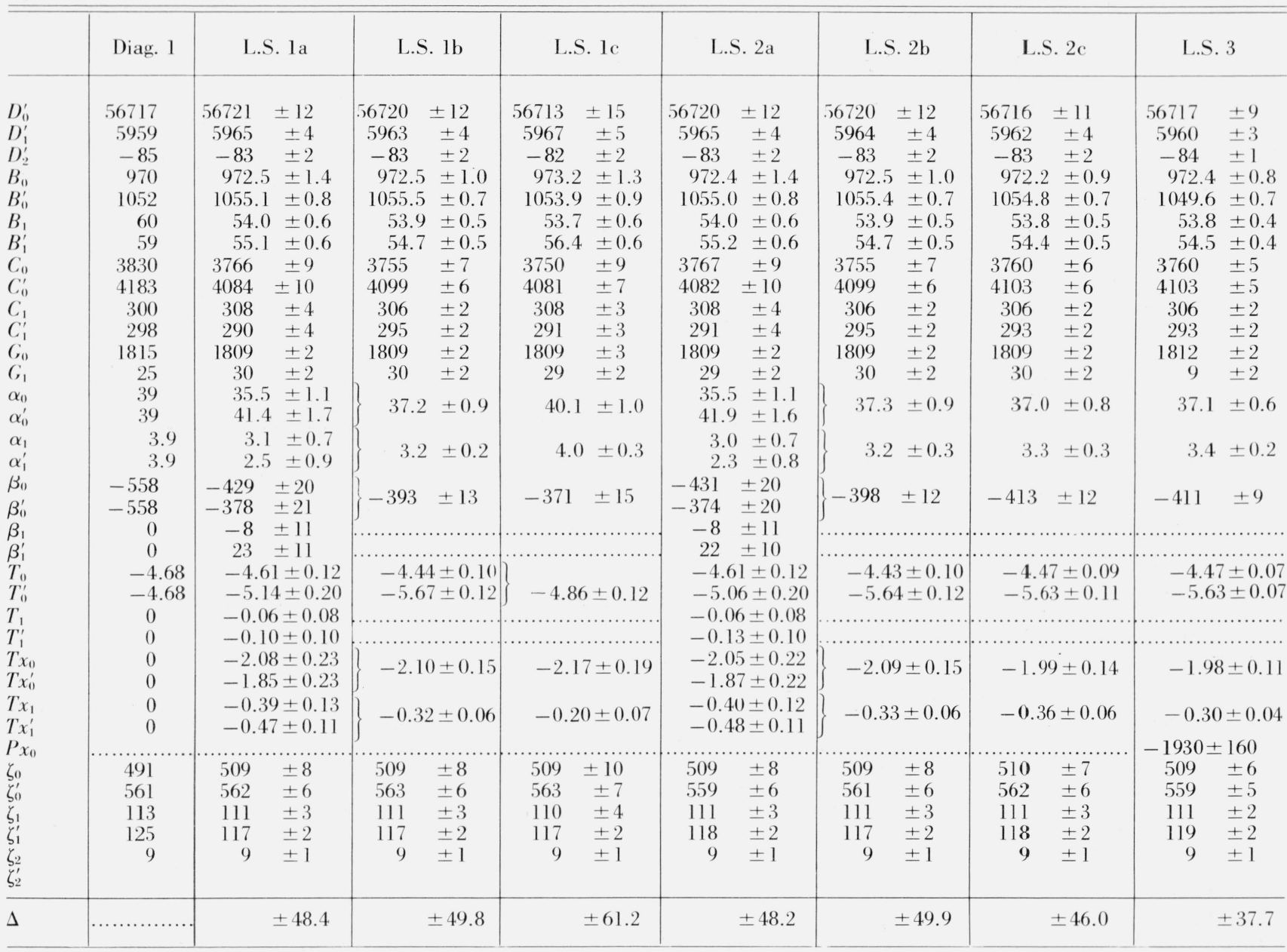

The parameter $P x$ is constant for all $d^{n-1} s$ configurations, so that its linear coefficient $P x_{1}$ is equal to zero.

same as those used in the previous work [3]; except that the above mentioned $a^{1} \mathrm{~S}$ of $\mathrm{Fe}$ III was also included.

In L.S. la, all of the 44 parameters were free, and the rms error is $48.4 \mathrm{~cm}^{-1}$. The parameters of this L.S. calculation are given in table 1 in the column headed L.S. la. A detailed examination of the results of this calculation shows that a great deal of unnecessary freedom was allowed to many of the parameters. Therefore, we performed L.S. Ib in which this extra freedom was eliminated by imposing the following restrictions on the parameters:

$$
\begin{aligned}
\alpha_{0}^{\prime} & =\alpha_{0} \\
\alpha_{1}^{\prime} & =\alpha_{1} \\
\beta_{0}^{\prime} & =\beta_{0} \\
\beta_{1}^{\prime} & =\beta_{1}=0 \\
T_{1}^{\prime} & =T_{1}=0 \\
T x_{0}^{\prime} & =T x_{0} \\
T x_{1}^{\prime} & =T x_{1}
\end{aligned}
$$

In this way, the number of free parameters was reduced from 44 to 35 . The rms error almost didn't increase, and it assumed the value of $49.8 \mathrm{~cm}^{-1}$. In L.S. 1c where, in addition, we also imposed the requirement $T_{0}=T_{0}^{\prime}$, the rms error increased to $61.2 \mathrm{~cm}^{-1}$. This means that these two parameters should be allowed to assume different values. In order to achieve convergence, we used the parameters of L.S. lb for a second diagonalization, Diag. II.

The derivatives of Diag. II were also used for three L.S. calculations designated as L.S. 2a, L.S. $2 \mathrm{~b}$ and L.S. 2c. In L.S. 2a, again, all the parameters were allowed to change freely and the rms error was 48.2 $\mathrm{cm}^{-1}$. In L.S. $2 \mathrm{~b}$, we imposed the same requirements upon the parameters as in L.S. $1 \mathrm{~b}$, and the rms error was $49.9 \mathrm{~cm}^{-1}$. The results of L.S. $2 \mathrm{a}$ and L.S. $2 \mathrm{~b}$ are practically equal to those of L.S. la and L.S. Ib, respectively, which means that mathematical convergence was achieved. In this stage of the calculation, we noted that the level $d^{7} s\left(b^{2} \mathrm{D}\right){ }^{3} \mathrm{D}_{1}$ of $\mathrm{Ni}$ III deviated from its calculated value by about $+300 \mathrm{~cm}^{-1}$, while the ${ }^{3} \mathrm{D}_{2}$ and ${ }^{3} \mathrm{D}_{3}$ of the same term had the deviations of -74 
$\mathrm{cm}^{-1}$ and $-52 \mathrm{~cm}^{-1}$, respectively. In L.S. $2 \mathrm{c}$, the same restrictions were imposed on the parameters as in L.S. $2 b$, but the above mentioned ${ }^{3} \mathrm{D}_{1}$ of $\mathrm{Ni}$ III was not included. The rms error was reduced to $46.0 \mathrm{~cm}^{-1}$. The deviations of the ${ }^{3} \mathrm{D}_{2}$ and ${ }^{3} \mathrm{D}_{3}$ were reduced to $-3 \mathrm{~cm}^{-1}$ and $-17 \mathrm{~cm}^{-1}$, respectively. The parameters didn't change within their uncertainties. The parameters of L.S. $2 \mathrm{a}, 2 \mathrm{~b}$, and $2 \mathrm{c}$ are given in table 1 under the corresponding columns. The fit between the observed and calculated levels in the above mentioned calculations is practically the same as in reference [3], except for the above mentioned $a^{1} \mathrm{~S}$ of Fe III which was reincluded in these calculations, and the ${ }^{3} \mathrm{D}_{1}$ of $\mathrm{Ni}$ III which was excluded from them.

\section{The Interaction Between the Configurations $3 d^{n}$ and $3 d^{n-1} 4 s$}

In the previous work [3], we could not determine the interaction between the configurations $d^{n}$ and $d^{n-1} s$. One should remember that this interaction is determined by one Slater parameter, $H$, also defined in reference [1]. Since, in its algebraic matrix, all the diagonal elements are equal to zero, it follows that if the initial value zero is given to the parameter $H$, the derivatives of all the eigen-values of the energy matrix with respect to $\mathrm{H}$ are also zero; and the leastsquares calculation, in which linear dependence of the levels upon the parameters is assumed, is not able to determine this parameter. Because of the above mentioned considerations, we tried, in the previous work, to assign various nonzero initial values to $H$, and to use the derivatives of the corresponding diagonalizations for making several least-squares calculations. In all of these calculations, $\mathrm{H}$ assumed unreasonable values (e.g., the value zero, or even changed its sign). In addition, the behavior of these values for the various spectra of the sequence was unsystematic. From these facts, we concluded that the actual interaction between the above mentioned configurations is so weak that, actually, $H$ was determined by accidental features of the experimental material in the spectra. Therefore, in the final stages of our previous calculation [3], we did not include the interaction between the above mentioned configurations in the Hamiltonian.

Since, in the present calculation, we achieved a rms error four times smaller than in the previous paper, which means a corresponding increase in the sensitivity of our least-squares calculation for weaker interactions, we decided to try to reinclude the interaction between $d^{n}$ and $d^{n-1}$ s in our calculation. We performed a diagonalization in which we used the values obtained in our analogous calculation on the second spectra of the iron group [1] as initial values for $H$. In the resulting least-squares calculation, we again obtained nonreasonable, nonsystematic values for $H$.

At this stage, we considered the possibility that our failure to determine a significant value fot the parameter $H$ was the result of our having ignored the inter- action of the configurations $d^{n}$ and $d^{n-1} \mathrm{~s}$ with the third configuration $d^{n-2} s^{2}$. Fortunately, Shenstone sent us new levels of Cu III belonging to the configuration $d^{7} s^{2}$. This enabled us to make a calculation in which the interactions of the configurations $d^{n-2} s^{2}$ were also included just by estimating a value for the linear coefficient in the interpolation formula of the parameter $D^{\prime \prime}$ (for its definition, see reference [1]). Unfortunately, even this more complete calculation didn't improve the meaningfulness of the results obtained for $H$.

Finally, we made a third effort. From the Ph.D. thesis of C. Roth [18], in which he calculated the configurations $d^{n} p$ and $d^{n-1} s p$ for various second spectra of the iron group, we were able to obtain some information about the value of the parameter $H$. In this case, the interaction between the above mentioned configurations is represented by three parameters:

$$
\begin{aligned}
H & =R^{2}(d d, d s) / 35 \\
J & =R^{2}(d p, s p) / 5 \\
K & =R^{1}(d p, p s) / 3
\end{aligned}
$$

$J$ and $K$ are much larger than $H$, and since they appear in the same nondiagonal elements of the energy matrix as does $H$, they also greatly increase the derivatives of the eigen-values with respect to $H$. Therefore, in the least-squares calculations performed by Roth, $H$ was determined. So, we performed a new diagonalization in which $H$ was given the values obtained in Roth's calculations. The derivatives of our new diagonalization were used for two least-squares calculations: In the first one, $H$ was free, and it consistently assumed the value zero. In the second one, $H$ was forced to maintain the value it was given in the diagonalization. This caused the rms error to increase from 46 to $51 \mathrm{~cm}^{-1}$.

Thus at this stage of the present calculations $H$ still remains undefined.

\section{The Effective Interactions Between $3 d$ and and $4 s$ Electrons}

In order to complete the process of describing all the perturbations of the configurations of the type $(d+s)^{n}$ by farlying perturbing configurations, one still has to consider that kind of effective-interactions which also include $s$ electrons. Since, the configuration $d s$ consists of only two terms, it is fully described by the two Slater parameters $F_{0}(d s)$ and $G_{2}(d s)$. Hence, it is evident that no additional two-body effective-interactions with $s$ electrons exist. On the other hand, three-body effective-interactions of such a kind are mathematically possible, and it is very easy to demonstrate that they can really appear in an actual physical Hamiltonian just by considering a possible actual perturbing configuration. By using grouptheoretical methods, Feneuille [15] was able to show that only five linearly independent three-body interaction operators can be added to the electrostatic Hamiltonian of a $(d+s)^{n}$ configuration, assuming that 
all the two-body effective-interactions have already been added to it.

Since in our previous calculations we already used the two-body effective-interactions represented by $\alpha$ and $\beta$, and the three-body interactions represented by $T$ and $T x$, we had to build three additional threebody operators. We did it in the following way: following Racah and Stein [13], let us define $u_{i}^{(k)}$ and $Y_{i}^{(k)}$, which are the one-electron tensorial operators of the order $k$ acting on the coordinates of the $i$ th electron, by their reduced matrices:

TABLE 2. Relative importance of the various effective interaction parameters

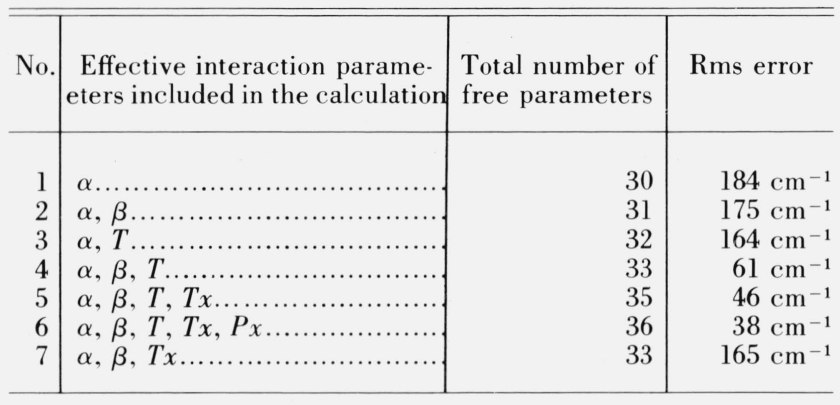

$$
\left(d|| u_{i}^{(k)}|| d\right)=1 ; \quad\left(d|| y_{i}^{(2)}|| s\right)=1 .
$$

All the other elements of the reduced matrices are equal to zero. Now we can define the operators

$$
U^{(k)}=\Sigma_{i} u_{i}^{(k)} ; \quad Y^{(2)}=\Sigma_{i} y_{i}^{(2)} .
$$

By using the method developed in the above mentioned paper of Racah and Stein [13], one can easily show that all of the second order perturbations caused to the $(d+s)^{n}$ configurations can be represented by a zero order product of such operators, or by suitable linear combinations of such products. We are not interested in products consisting of $U^{(k)}$ 's only, since interactions between only $d$ electrons are already completely described. Thus the operators representing new interactions may be of the forms

$$
\begin{aligned}
& {\left[U^{(k)} \times Y^{(2)} \times Y^{(2)} \dagger\right]^{(0)}+\text { h.c. }} \\
& {\left[U^{(k)} \times U^{\left(k^{\prime}\right)} \times Y^{(2)}\right]^{(0)}+\text { h.c. }} \\
& {\left[U^{(k)} \times Y^{(2)} \times Y^{(2)}\right]^{(0)}+\text { h.c. }}
\end{aligned}
$$

where "h.c." means "hermitian conjugate."

The first of these operators acts within the configuration $d^{n-1} s$. The second one contributes to the interaction between $d^{n}$ and $d^{n-1} s$ or between $d^{n-1} s$ and $d^{n-2} s^{2}$. The third contributes to the interaction between the configurations $d^{n}$ and $d^{n-2} s^{2}$.

An appropriate set of operators of the above mentioned types can be chosen by considering the configuration $(d+s)^{3}$. A simple counting of the terms of the configuration $d^{2} s$ and of the nonzero, nondiagonal elements connecting the configurations $d^{3}$ and $d s^{2}$ shows that we have exactly one independent threebody operator acting within $d^{2} s$, and one such operator connecting the configurations $d^{3}$ and $d s^{2}$. Thus the third independent operator must connect the configurations $d^{3}$ and $d^{2} s$. It turns out that we have to add to our hamiltonian one operator of each of the above mentioned types. Since there are no more independent operators, it is unimportant if, in the formulas (1), (2), and (3), we set $k$ and $k^{\prime}$ equal to 2 or 4. Thus we completed the definition of the operators by arbitrarily choosing for $k$ and $k^{\prime}$ the value 2 . Then we defined three parameters, $P x, P y$, and $P z$ which are the coefficients of the above mentioned operators numbered as 1,2 , and 3 , respectively. Practically, $P x, P y$, and $P z$ are three additional correction-terms whose numerical values may be determined by the usual least-squares calculation method.

In our previous calculations, we could not include even the actual electrostatic interaction between the configurations $d^{n}$ and $d^{n-1} s$. Hence, there is no hope that a much smaller correction of this interaction, represented by the parameter $P y$, will have any detectable effect. Thus, it seemed reasonable to introduce only the new effective-interaction parameter $P x$ which "acts" within the configuration $d^{n-1} s$. In the column "L.S. 3" of table 1, the results of its introduction are reported. We see that the addition of only one parameter has reduced the rms error from 46 to $38 \mathrm{~cm}^{-1}$. The new parameter is well defined. This is demonstrated by the fact that its numerical value is considerably larger than its corresponding uncertainty. It should be noted that at this final stage of the calculation we included 12 additional levels of Co III, which had been observed by A. G. Shenstone [19]. Thus, the total number of observed levels which were fitted to the calculated ones is 334 . The observed and calculated levels as calculated in L.S. 3 are reported in tables 4 to 13 . 
TABLE 3. Comparison of the configurations $3 \mathrm{~d}^{\mathrm{n}}$ and $3 \mathrm{~d}^{\mathrm{n}-1} 4 \mathrm{~s}$ for the various approximations

\begin{tabular}{l|r|r}
\hline $\begin{array}{c}\text { Effective-interaction } \\
\text { parameters included in } \\
\text { the calculation }\end{array}$ & $\begin{array}{c}\text { Rms-error for } \\
\text { the obs. levels } \\
\text { of } 3 d^{n}\end{array}$ & $\begin{array}{c}\text { Rms-error for } \\
\text { the obs. levels } \\
\text { of } 3 d^{n-1} 4 s\end{array}$ \\
\hline$\alpha$ & $233 \mathrm{~cm}^{-1}$ & $136 \mathrm{~cm}^{-1}$ \\
$\alpha, \beta, T$ & $59 \mathrm{~cm}^{-1}$ & $60 \mathrm{~cm}^{-1}$ \\
$\alpha, \beta, T, T x$ & $35 \mathrm{~cm}^{-1}$ & $32 \mathrm{~cm}^{-1}$ \\
$\alpha, \beta, T, T x, P x$ & $35 \mathrm{~cm}^{-1}$ & \\
\hline
\end{tabular}

TABLE 4. Sc III-observed and calculated energy levels

\begin{tabular}{l|c|r|r|r|r|r}
\hline \hline \multirow{2}{*}{ CONF. } & TERM & $J$ & OBS. & CALC. & O-C & CALC. $g$ \\
\hline \multirow{2}{*}{$s$} & \multirow{2}{*}{${ }^{2} \mathrm{D}$} & $3 / 2$ & 0.0 & -97 & 97 & 0.800 \\
& & $5 / 2$ & 197.5 & 130 & 68 & 1.200 \\
& \multirow{2}{*}{${ }^{2} \mathrm{~S}$} & $1 / 2$ & 25536.7 & 25701 & -164 & 2.000 \\
\hline
\end{tabular}

TABLE 5. Ti III-observed and calculated energy levels

\begin{tabular}{|c|c|c|c|c|c|c|}
\hline CONF. & TERM & $J$ & OBS. & CALC. & $\mathrm{O}-\mathrm{C}$ & CALC. $g$ \\
\hline$d^{2}$ & ${ }^{3} \mathrm{~F}$ & $\begin{array}{l}2 \\
3 \\
4\end{array}$ & $\begin{array}{r}0.0 \\
183.7 \\
421.9\end{array}$ & $\begin{array}{r}-34 \\
150 \\
390\end{array}$ & $\begin{array}{l}34 \\
34 \\
32\end{array}$ & $\begin{array}{l}0.667 \\
1.083 \\
1.250\end{array}$ \\
\hline$d^{2}$ & ${ }^{1} \mathrm{D}$ & 2 & 8472.6 & 8456 & 17 & 1.002 \\
\hline$d^{2}$ & ${ }^{3} \mathrm{P}$ & $\begin{array}{l}0 \\
1 \\
2\end{array}$ & $\begin{array}{l}10536.4 \\
10603.5 \\
10721.1\end{array}$ & $\begin{array}{l}10546 \\
10611 \\
10744\end{array}$ & $\begin{array}{r}-10 \\
-8 \\
-23\end{array}$ & $\begin{array}{l}0.000 \\
1.500 \\
1.497\end{array}$ \\
\hline$d^{2}$ & ${ }^{1} \mathrm{G}$ & 4 & 14398.5 & 14404 & -6 & 1.000 \\
\hline$d^{2}$ & ${ }^{1} \mathrm{~S}$ & 0 & & 32480 & & 0.000 \\
\hline$d s$ & $\left({ }^{2} \mathrm{D}\right)^{3} \mathrm{D}$ & $\begin{array}{l}1 \\
2 \\
3\end{array}$ & $\begin{array}{l}38063.50 \\
38197.98 \\
38425.19\end{array}$ & $\begin{array}{l}38096 \\
38224 \\
38436\end{array}$ & $\begin{array}{l}-33 \\
-26 \\
-11\end{array}$ & $\begin{array}{l}0.500 \\
1.166 \\
1.333\end{array}$ \\
\hline$d s$ & $\left({ }^{2} \mathrm{D}\right)^{1} \mathrm{D}$ & 2 & 41703.65 & 41705 & -1 & 1.000 \\
\hline
\end{tabular}

TABLE 6. V III-observed and calculated energy levels

\begin{tabular}{|c|c|c|c|c|c|c|}
\hline CONF. & TERM & $J$ & OBS. & CALC. & $\mathrm{O}-\mathrm{C}$ & CALC. $g$ \\
\hline$d^{3}$ & ${ }^{4} \mathrm{~F}$ & $\begin{array}{l}3 / 2 \\
5 / 2 \\
7 / 2 \\
9 / 2\end{array}$ & $\begin{array}{r}0.0 \\
145.5 \\
341.5 \\
583.8\end{array}$ & $\begin{array}{r}34 \\
178 \\
375 \\
622\end{array}$ & $\begin{array}{l}-34 \\
-33 \\
-34 \\
-38\end{array}$ & $\begin{array}{l}0.400 \\
1.029 \\
1.238 \\
1.333\end{array}$ \\
\hline$d^{3}$ & ${ }^{4} \mathrm{P}$ & $\begin{array}{l}1 / 2 \\
3 / 2 \\
5 / 2\end{array}$ & $\begin{array}{l}11513.8 \\
11591.8 \\
11769.7\end{array}$ & $\begin{array}{l}11544 \\
11610 \\
11773\end{array}$ & $\begin{array}{r}-30 \\
-18 \\
-3\end{array}$ & $\begin{array}{l}2.661 \\
1.730 \\
1.600\end{array}$ \\
\hline$d^{3}$ & ${ }^{2} \mathrm{G}$ & $\begin{array}{l}7 / 2 \\
9 / 2\end{array}$ & $\begin{array}{l}11966.3 \\
12187.0\end{array}$ & $\begin{array}{l}11989 \\
12205\end{array}$ & $\begin{array}{l}-23 \\
-18\end{array}$ & $\begin{array}{l}0.889 \\
1.111\end{array}$ \\
\hline$d^{3}$ & ${ }^{2} \mathrm{P}$ & $\begin{array}{l}3 / 2 \\
1 / 2\end{array}$ & $\begin{array}{l}15550.3 \\
15579.8\end{array}$ & $\begin{array}{l}15563 \\
15600\end{array}$ & $\begin{array}{l}-13 \\
-20\end{array}$ & $\begin{array}{l}1.182 \\
0.672\end{array}$ \\
\hline$d^{3}$ & $a^{2} \mathrm{D}$ & $\begin{array}{l}3 / 2 \\
5 / 2\end{array}$ & $\begin{array}{l}16330.5 \\
16374.7\end{array}$ & $\begin{array}{l}16308 \\
16349\end{array}$ & $\begin{array}{l}23 \\
26\end{array}$ & $\begin{array}{l}0.954 \\
1.200\end{array}$ \\
\hline$d^{3}$ & ${ }^{2} \mathrm{H}$ & $\begin{array}{r}9 / 2 \\
11 / 2\end{array}$ & $\begin{array}{l}16810.9 \\
16977.6\end{array}$ & $\begin{array}{l}16831 \\
17000\end{array}$ & $\begin{array}{l}-20 \\
-22\end{array}$ & $\begin{array}{l}0.910 \\
1.091\end{array}$ \\
\hline$d^{3}$ & ${ }^{2} \mathrm{~F}$ & $\begin{array}{l}7 / 2 \\
5 / 2\end{array}$ & $\begin{array}{l}27727.8 \\
27846.8\end{array}$ & $\begin{array}{l}27743 \\
27835\end{array}$ & $\begin{array}{r}-15 \\
12\end{array}$ & $\begin{array}{l}1.143 \\
0.857\end{array}$ \\
\hline$d^{3}$ & $b^{2} \mathrm{D}$ & $\begin{array}{l}5 / 2 \\
3 / 2\end{array}$ & $\begin{array}{l}42267.4 \\
42371.2\end{array}$ & $\begin{array}{l}42233 \\
42352\end{array}$ & $\begin{array}{l}34 \\
19\end{array}$ & $\begin{array}{l}1.200 \\
0.800\end{array}$ \\
\hline$d^{2} s$ & $\left({ }^{3} \mathrm{~F}\right)^{4} \mathrm{~F}$ & $\begin{array}{l}3 / 2 \\
5 / 2 \\
7 / 2 \\
9 / 2\end{array}$ & $\begin{array}{l}43942.49 \\
44110.04 \\
44345.84 \\
44646.96\end{array}$ & $\begin{array}{l}43945 \\
44104 \\
44330 \\
44621\end{array}$ & $\begin{array}{r}-3 \\
6 \\
16 \\
26\end{array}$ & $\begin{array}{l}0.400 \\
1.028 \\
1.238 \\
1.333\end{array}$ \\
\hline$d^{2} s$ & $\left({ }^{3} \mathrm{~F}\right)^{2} \mathrm{~F}$ & $\begin{array}{l}5 / 2 \\
7 / 2\end{array}$ & $\begin{array}{l}49327.74 \\
49805.29\end{array}$ & $\begin{array}{l}49332 \\
49786\end{array}$ & $\begin{array}{r}-4 \\
19\end{array}$ & $\begin{array}{l}0.858 \\
1.143\end{array}$ \\
\hline$d^{2} s$ & $\left({ }^{1} \mathrm{D}\right)^{2} \mathrm{D}$ & $\begin{array}{l}5 / 2 \\
3 / 2\end{array}$ & $\begin{array}{l}56160.42 \\
56256.75\end{array}$ & $\begin{array}{l}56148 \\
56224\end{array}$ & $\begin{array}{l}12 \\
33\end{array}$ & $\begin{array}{l}1.262 \\
0.864\end{array}$ \\
\hline$d^{2} s$ & $\left({ }^{3} \mathrm{P}\right)^{4} \mathrm{P}$ & $\begin{array}{l}1 / 2 \\
3 / 2 \\
5 / 2\end{array}$ & $\begin{array}{l}56529.7 \\
56669.2 \\
56922.7\end{array}$ & $\begin{array}{l}56526 \\
56657 \\
56908\end{array}$ & $\begin{array}{r}4 \\
12 \\
15\end{array}$ & $\begin{array}{l}2.666 \\
1.671 \\
1.537\end{array}$ \\
\hline$d^{2} s$ & $\left({ }^{3} \mathrm{P}\right)^{2} \mathrm{P}$ & $\begin{array}{l}1 / 2 \\
3 / 2\end{array}$ & $\begin{array}{l}61578.74 \\
61777.15\end{array}$ & $\begin{array}{l}61569 \\
61776\end{array}$ & $\begin{array}{r}10 \\
1\end{array}$ & $\begin{array}{l}0.667 \\
1.332\end{array}$ \\
\hline$d^{2} s$ & $\left({ }^{1} \mathrm{G}\right)^{2} \mathrm{G}$ & $\begin{array}{l}7 / 2 \\
9 / 2\end{array}$ & $\begin{array}{l}63304.64 \\
63313.90\end{array}$ & $\begin{array}{l}63268 \\
63268\end{array}$ & $\begin{array}{l}37 \\
46\end{array}$ & $\begin{array}{l}0.889 \\
1.111\end{array}$ \\
\hline$d^{2} s$ & $\left({ }^{1} \mathrm{~S}\right)^{2} \mathrm{~S}$ & $1 / 2$ & & 86152 & & 2.000 \\
\hline
\end{tabular}


TABLE 7. Cr III-observed and calculated energy levels

\begin{tabular}{|c|c|c|c|c|c|c|}
\hline CONF. & TERM & $J$ & OBS. & CALC. & $\mathrm{O}-\mathrm{C}$ & CALC. $g$ \\
\hline$d^{4}$ & ${ }^{5} \mathrm{D}$ & $\begin{array}{l}0 \\
1 \\
2 \\
3 \\
4\end{array}$ & $\begin{array}{r}0.0 \\
59.9 \\
181.9 \\
355.8 \\
575.0\end{array}$ & $\begin{array}{r}-20 \\
42 \\
164 \\
342 \\
570\end{array}$ & $\begin{array}{r}20 \\
18 \\
18 \\
14 \\
5\end{array}$ & $\begin{array}{l}0.000 \\
1.500 \\
1.500 \\
1.500 \\
1.500\end{array}$ \\
\hline$d^{4}$ & $a^{3} \mathrm{P}$ & $\begin{array}{l}0 \\
1 \\
2\end{array}$ & $\begin{array}{l}16770.9 \\
17167.4 \\
17850.0\end{array}$ & $\begin{array}{l}16788 \\
17170 \\
17850\end{array}$ & $\begin{array}{r}-17 \\
-3 \\
-0\end{array}$ & $\begin{array}{l}0.000 \\
1.499 \\
1.500\end{array}$ \\
\hline$d^{4}$ & ${ }^{3} \mathrm{H}$ & $\begin{array}{l}4 \\
5 \\
6\end{array}$ & $\begin{array}{l}17272.8 \\
17395.5 \\
17528.8\end{array}$ & $\begin{array}{l}17297 \\
17431 \\
17583\end{array}$ & $\begin{array}{l}-24 \\
-36 \\
-54\end{array}$ & $\begin{array}{l}0.802 \\
1.034 \\
1.166\end{array}$ \\
\hline$d^{4}$ & $a^{3} \mathrm{~F}$ & $\begin{array}{l}2 \\
3 \\
4\end{array}$ & $\begin{array}{l}18451.0 \\
18510.0 \\
18581.6\end{array}$ & $\begin{array}{l}18460 \\
18519 \\
18598\end{array}$ & $\begin{array}{r}-9 \\
-9 \\
-16\end{array}$ & $\begin{array}{l}0.667 \\
1.078 \\
1.245\end{array}$ \\
\hline$d^{4}$ & ${ }^{3} \mathrm{G}$ & $\begin{array}{l}3 \\
4 \\
5\end{array}$ & $\begin{array}{l}20702.0 \\
20851.3 \\
20994.6\end{array}$ & $\begin{array}{l}20741 \\
20900 \\
21049\end{array}$ & $\begin{array}{l}-39 \\
-49 \\
-54\end{array}$ & $\begin{array}{l}0.755 \\
1.052 \\
1.199\end{array}$ \\
\hline$d^{4}$ & $a^{1} \mathrm{G}$ & 4 & & 25092 & & 1.001 \\
\hline$d^{4}$ & ${ }^{3} \mathrm{D}$ & $\begin{array}{l}3 \\
2 \\
1\end{array}$ & $\begin{array}{l}25725.8 \\
25781.0 \\
25848.2\end{array}$ & $\begin{array}{l}25741 \\
25794 \\
25848\end{array}$ & $\begin{array}{r}-15 \\
-13 \\
0\end{array}$ & $\begin{array}{l}1.333 \\
1.167 \\
0.501\end{array}$ \\
\hline$d^{4}$ & ${ }^{1} \mathrm{I}$ & 6 & & 25981 & & 1.000 \\
\hline$d^{4}$ & $a^{\prime} S$ & 0 & & 27289 & & 0.000 \\
\hline$d^{4}$ & $a^{1} \mathrm{D}$ & 2 & & 32065 & & 1.000 \\
\hline$d^{4}$ & ${ }^{1} \mathrm{~F}$ & 3 & & 36938 & & 1.000 \\
\hline$d^{4}$ & $b^{3} \mathrm{~F}$ & $\begin{array}{l}4 \\
3 \\
2\end{array}$ & $\begin{array}{l}43286.4 \\
43321.7 \\
43304.1\end{array}$ & $\begin{array}{l}43281 \\
43332 \\
43334\end{array}$ & $\begin{array}{r}5 \\
-10 \\
-30\end{array}$ & $\begin{array}{l}1.250 \\
1.083 \\
0.684\end{array}$ \\
\hline$d^{4}$ & $b^{3} \mathrm{P}$ & $\begin{array}{l}2 \\
1 \\
0\end{array}$ & & $\begin{array}{l}43344 \\
43801 \\
44030\end{array}$ & & $\begin{array}{l}1.482 \\
1.500 \\
0.000\end{array}$ \\
\hline$d^{4}$ & $b^{1} \mathrm{G}$ & 4 & & 49594 & & 1.000 \\
\hline$d^{3} s$ & $\left({ }^{4} \mathrm{~F}\right)^{5} \mathrm{~F}$ & $\begin{array}{l}1 \\
2 \\
3 \\
4 \\
5\end{array}$ & $\begin{array}{l}49491.0 \\
49626.8 \\
49827.7 \\
50089.2 \\
50408.2\end{array}$ & $\begin{array}{l}49479 \\
49615 \\
49817 \\
50084 \\
50413\end{array}$ & $\begin{array}{r}12 \\
12 \\
11 \\
5 \\
-5\end{array}$ & $\begin{array}{l}0.000 \\
1.000 \\
1.250 \\
1.350 \\
1.400\end{array}$ \\
\hline$d^{3} s$ & $\left({ }^{4} \mathrm{~F}\right)^{3} \mathrm{~F}$ & $\begin{array}{l}2 \\
3 \\
4\end{array}$ & $\begin{array}{l}56650.5 \\
56992.2 \\
57422.1\end{array}$ & $\begin{array}{l}56658 \\
56998 \\
57435\end{array}$ & $\begin{array}{r}-8 \\
-6 \\
-13\end{array}$ & $\begin{array}{l}0.667 \\
1.083 \\
1.250\end{array}$ \\
\hline$d^{3} s$ & $\left({ }^{4} \mathrm{P}\right)^{5} \mathrm{P}$ & $\begin{array}{l}1 \\
2 \\
3\end{array}$ & $\begin{array}{l}63038.6 \\
63172.2 \\
63420.3\end{array}$ & $\begin{array}{l}63052 \\
63167 \\
63396\end{array}$ & $\begin{array}{r}-13 \\
5 \\
24\end{array}$ & $\begin{array}{l}2.497 \\
1.831 \\
1.666\end{array}$ \\
\hline$d^{4}$ & $b^{1} \mathrm{D}$ & 2 & & 65585 & & 1.000 \\
\hline$d^{3} s$ & $\left({ }^{2} \mathrm{G}\right)^{3} \mathrm{G}$ & $\begin{array}{l}3 \\
4 \\
5\end{array}$ & $\begin{array}{l}65890.9 \\
66027.8 \\
66222.7\end{array}$ & $\begin{array}{l}65855 \\
65997 \\
66196\end{array}$ & $\begin{array}{l}36 \\
31 \\
27\end{array}$ & $\begin{array}{l}0.750 \\
1.049 \\
1.199\end{array}$ \\
\hline$d^{3} s$ & $\left({ }^{2} \mathrm{G}\right)^{1} \mathrm{G}$ & 4 & & 69622 & & 0.994 \\
\hline$d^{3} s$ & $\begin{array}{l}60 \%\left({ }^{4} \mathrm{P}\right)+40 \%\left({ }^{2} \mathrm{P}\right)^{3} \mathrm{P} \\
58 \%\left({ }^{6} \mathrm{P}\right)^{3} \mathrm{P}+34 \%\left({ }^{2} \mathrm{P}\right)^{3} \mathrm{P} \\
70 \%\left({ }^{4} \mathrm{P}\right)^{3} \mathrm{P}+13 \%\left({ }^{2} \mathrm{P}\right)^{3} \mathrm{P}\end{array}$ & $\begin{array}{l}0 \\
1 \\
2\end{array}$ & $\begin{array}{l}69510.6 \\
69780.9 \\
70291.8\end{array}$ & $\begin{array}{l}69594 \\
69764 \\
70247\end{array}$ & $\begin{array}{r}-83 \\
17 \\
45\end{array}$ & $\begin{array}{l}0.000 \\
1.422 \\
1.475\end{array}$ \\
\hline
\end{tabular}


TABLE 7. CrIII-observed and calculated energy levels-Continued

\begin{tabular}{|c|c|c|c|c|c|c|}
\hline CONF. & TERM & $J$ & OBS. & CALC. & $\mathrm{O}-\mathrm{C}$ & CALC. $g$ \\
\hline$d^{3} \mathrm{~s}$ & $\begin{array}{l}60 \%\left({ }^{2} \mathrm{P}\right)^{3} \mathrm{P}+40 \%\left({ }^{4} \mathrm{P}\right)^{3} \mathrm{P} \\
55 \%\left({ }^{2} \mathrm{P}\right)^{3} \mathrm{P}+40 \%\left({ }^{6} \mathrm{P}\right)^{3} \mathrm{P} \\
86 \%\left({ }^{2} \mathrm{P}\right)^{3} \mathrm{P}+7 \%\left({ }^{4} \mathrm{P}\right)^{3} \mathrm{P}\end{array}$ & $\begin{array}{l}2 \\
1 \\
0\end{array}$ & & $\begin{array}{l}70155 \\
70306 \\
70445\end{array}$ & & $\begin{array}{l}1.445 \\
1.423 \\
0.000\end{array}$ \\
\hline$d^{3} s$ & $\left(a^{2} \mathrm{D}\right)^{3} \mathrm{D}$ & $\begin{array}{l}1 \\
2 \\
3\end{array}$ & $\begin{array}{l}70980.2 \\
71166.1 \\
71321.8\end{array}$ & $\begin{array}{l}70924 \\
71255 \\
71269\end{array}$ & $\begin{array}{r}56 \\
-89 \\
53\end{array}$ & $\begin{array}{l}0.659 \\
1.245 \\
1.333\end{array}$ \\
\hline$d^{3} s$ & $\left({ }^{2} \mathrm{H}\right)^{3} \mathrm{H}$ & $\begin{array}{l}4 \\
5\end{array}$ & $\begin{array}{l}71676.2 \\
71736.0\end{array}$ & $\begin{array}{l}71602 \\
71677\end{array}$ & $\begin{array}{l}74 \\
59\end{array}$ & $\begin{array}{l}0.807 \\
1.034\end{array}$ \\
\hline & & 6 & 71868.5 & 71822 & 47 & 1.167 \\
\hline$d^{3} s$ & $\left({ }^{2} \mathrm{P}\right)^{1} \mathrm{P}$ & 1 & & 73886 & & 0.998 \\
\hline$d^{3} s$ & $\left(a^{2} \mathrm{D}\right)^{1} \mathrm{D}$ & 2 & & 74678 & & 1.004 \\
\hline$d^{3} s$ & $\left({ }^{2} \mathrm{H}\right)^{1} \mathrm{H}$ & 5 & & 75314 & & 1.000 \\
\hline$d^{4}$ & $b{ }^{1} \mathrm{~S}$ & 0 & & 83901 & & 0.000 \\
\hline$d^{3} s$ & $\left({ }^{2} \mathrm{~F}\right)^{3} \mathrm{~F}$ & $\begin{array}{l}4 \\
3 \\
2\end{array}$ & & $\begin{array}{l}84316 \\
84396 \\
84460\end{array}$ & & $\begin{array}{l}1.250 \\
1.083 \\
0.667\end{array}$ \\
\hline$d^{3} s$ & $\left({ }^{2} \mathrm{~F}\right)^{1} \mathrm{~F}$ & 3 & & 87723 & & 1.000 \\
\hline$d^{3} s$ & $\left(b^{2} \mathrm{D}\right)^{3} \mathrm{D}$ & $\begin{array}{l}3 \\
2 \\
1\end{array}$ & & $\begin{array}{l}102089 \\
102189 \\
102259\end{array}$ & & $\begin{array}{l}1.333 \\
1.166 \\
0.500\end{array}$ \\
\hline$d^{3} s$ & $\left(b^{2} \mathrm{D}\right)^{1} \mathrm{D}$ & 2 & & 105516 & & 1.000 \\
\hline
\end{tabular}

TABLE 8. Mn III-observed and calculated energy levels

\begin{tabular}{|c|c|c|c|c|c|c|}
\hline CONF. & TERM & $J$ & OBS. & CALC. & $\mathrm{O}-\mathrm{C}$ & CALC. $g$ \\
\hline$d^{5}$ & ${ }^{6} \mathrm{~S}$ & $5 / 2$ & 0.0 & -188 & 188 & 2.000 \\
\hline$d^{5}$ & ${ }^{4} \mathrm{G}$ & $\begin{array}{r}11 / 2 \\
9 / 2 \\
7 / 2 \\
5 / 2\end{array}$ & $\begin{array}{l}26824.5 \\
26852.4 \\
26860.3 \\
26856.9\end{array}$ & $\begin{array}{l}26858 \\
26863 \\
26853 \\
26836\end{array}$ & $\begin{array}{r}-34 \\
-11 \\
7 \\
21\end{array}$ & $\begin{array}{l}1.273 \\
1.172 \\
0.984 \\
0.572\end{array}$ \\
\hline$d^{5}$ & ${ }^{4} \mathrm{P}$ & $\begin{array}{l}5 / 2 \\
3 / 2 \\
1 / 2\end{array}$ & $\begin{array}{l}29168.9 \\
29243.0\end{array}$ & $\begin{array}{l}29194 \\
29234 \\
29274\end{array}$ & $\begin{array}{r}-25 \\
9\end{array}$ & $\begin{array}{l}1.593 \\
1.722 \\
2.650\end{array}$ \\
\hline$d^{5}$ & ${ }^{4} \mathrm{D}$ & $\begin{array}{l}7 / 2 \\
5 / 2 \\
3 / 2 \\
1 / 2\end{array}$ & $\begin{array}{l}32308.9 \\
32385.7\end{array}$ & $\begin{array}{l}32324 \\
32401 \\
32395 \\
32367\end{array}$ & $\begin{array}{l}-15 \\
-15\end{array}$ & $\begin{array}{l}1.428 \\
1.376 \\
1.210 \\
0.017\end{array}$ \\
\hline$d^{5}$ & ${ }^{2} \mathbf{I}$ & $\begin{array}{l}11 / 2 \\
13 / 2\end{array}$ & & $\begin{array}{l}39144 \\
39188\end{array}$ & & $\begin{array}{l}0.924 \\
1.077\end{array}$ \\
\hline$d^{5}$ & $\begin{array}{l}77 \% a^{2} \mathrm{D}+22 \% a^{2} \mathrm{~F} \\
a^{2} \mathrm{D}\end{array}$ & $\begin{array}{l}5 / 2 \\
3 / 2\end{array}$ & & $\begin{array}{l}41199 \\
41543\end{array}$ & & $\begin{array}{l}1.124 \\
0.788\end{array}$ \\
\hline$d^{5}$ & $\begin{array}{l}\mathrm{a}^{2} \mathrm{~F} \\
75 \% a^{2} \mathrm{~F}+15 \% a^{2} \mathrm{D}\end{array}$ & $\begin{array}{l}7 / 2 \\
5 / 2\end{array}$ & & $\begin{array}{l}42583 \\
43064\end{array}$ & & $\begin{array}{l}1.143 \\
0.933\end{array}$ \\
\hline$d^{5}$ & ${ }^{4} \mathrm{~F}$ & $\begin{array}{l}9 / 2 \\
7 / 2 \\
5 / 2 \\
3 / 2\end{array}$ & $\begin{array}{l}43574.2 \\
43604.2 \\
43670.5 \\
43675.6\end{array}$ & $\begin{array}{l}43650 \\
43679 \\
43740 \\
43762\end{array}$ & $\begin{array}{l}-76 \\
-75 \\
-70 \\
-86\end{array}$ & $\begin{array}{l}1.331 \\
1.237 \\
1.030 \\
0.413\end{array}$ \\
\hline
\end{tabular}


TABLE 8. Mn III-observed and calculated energy levels-Continued

\begin{tabular}{|c|c|c|c|c|c|c|}
\hline CONF. & TERM & $J$ & OBS. & CALC. & $\mathrm{O}-\mathrm{C}$ & CALC. $g$ \\
\hline$d^{5}$ & ${ }^{2} \mathrm{H}$ & $\begin{array}{r}9 / 2 \\
11 / 2\end{array}$ & & $\begin{array}{l}46477 \\
46648\end{array}$ & & $\begin{array}{l}0.926 \\
1.090\end{array}$ \\
\hline$d^{5}$ & $a^{2} \mathrm{G}$ & $\begin{array}{l}7 / 2 \\
9 / 2\end{array}$ & & $\begin{array}{l}47806 \\
47954\end{array}$ & & $\begin{array}{l}0.890 \\
1.097\end{array}$ \\
\hline$d^{5}$ & $b^{2} \mathrm{~F}$ & $\begin{array}{l}5 / 2 \\
7 / 2\end{array}$ & & $\begin{array}{l}50938 \\
51013\end{array}$ & & $\begin{array}{l}0.858 \\
1.143\end{array}$ \\
\hline$d^{5}$ & ${ }^{2} \mathrm{~S}$ & $1 / 2$ & & 55616 & & 2.000 \\
\hline$d^{5}$ & $b^{2} \mathrm{D}$ & $\begin{array}{l}3 / 2 \\
5 / 2\end{array}$ & & $\begin{array}{l}61466 \\
61521\end{array}$ & & $\begin{array}{l}0.800 \\
1.199\end{array}$ \\
\hline$d^{4} s$ & $\left({ }^{5} \mathrm{D}\right)^{6} \mathrm{D}$ & $\begin{array}{l}1 / 2 \\
3 / 2 \\
5 / 2 \\
7 / 2 \\
9 / 2\end{array}$ & $\begin{array}{l}62456.7 \\
62567.9 \\
62747.3 \\
62988.9 \\
63285.2\end{array}$ & $\begin{array}{l}62427 \\
62541 \\
62727 \\
62980 \\
63295\end{array}$ & $\begin{array}{r}30 \\
27 \\
20 \\
9 \\
-10\end{array}$ & $\begin{array}{l}3.332 \\
1.866 \\
1.657 \\
1.587 \\
1.555\end{array}$ \\
\hline$d^{5}$ & $b^{2} \mathrm{G}$ & $\begin{array}{l}9 / 2 \\
7 / 2\end{array}$ & & $\begin{array}{l}68802 \\
68821\end{array}$ & & $\begin{array}{l}1.111 \\
0.889\end{array}$ \\
\hline$d^{4} s$ & $\left({ }^{5} \mathrm{D}\right)^{4} \mathrm{D}$ & $\begin{array}{l}1 / 2 \\
3 / 2 \\
5 / 2 \\
7 / 2\end{array}$ & $\begin{array}{l}71396.5 \\
71565.5 \\
71833.4 \\
72184.8\end{array}$ & $\begin{array}{l}71359 \\
71532 \\
71809 \\
72177\end{array}$ & $\begin{array}{r}38 \\
34 \\
24 \\
8\end{array}$ & $\begin{array}{l}0.001 \\
1.200 \\
1.371 \\
1.428\end{array}$ \\
\hline$d^{5}$ & ${ }^{2} \mathrm{P}$ & $\begin{array}{l}3 / 2 \\
1 / 2\end{array}$ & & $\begin{array}{l}83144 \\
83172\end{array}$ & & $\begin{array}{l}1.332 \\
0.667\end{array}$ \\
\hline$d^{4} s$ & $\left(a^{3} \mathrm{P}\right)^{4} \mathrm{P}$ & $\begin{array}{l}1 / 2 \\
3 / 2 \\
5 / 2\end{array}$ & & $\begin{array}{l}84598 \\
85154 \\
86043\end{array}$ & & $\begin{array}{l}2.659 \\
1.731 \\
1.599\end{array}$ \\
\hline$d^{4} s$ & $\left({ }^{3} \mathrm{H}\right)^{4} \mathrm{H}$ & $\begin{array}{r}7 / 2 \\
9 / 2 \\
11 / 2 \\
13 / 2\end{array}$ & & $\begin{array}{l}84934 \\
85048 \\
85195 \\
85373\end{array}$ & & $\begin{array}{l}0.670 \\
0.972 \\
1.134 \\
1.231\end{array}$ \\
\hline$d^{4} s$ & $\left(a^{3} \mathrm{~F}\right)^{4} \mathrm{~F}$ & $\begin{array}{l}3 / 2 \\
5 / 2 \\
7 / 2 \\
9 / 2\end{array}$ & & $\begin{array}{l}86463 \\
86501 \\
86566 \\
86658\end{array}$ & & $\begin{array}{l}0.401 \\
1.021 \\
1.230 \\
1.327\end{array}$ \\
\hline$d^{4} s$ & $\left({ }^{3} \mathrm{G}\right)^{4} \mathrm{G}$ & $\begin{array}{r}5 / 2 \\
7 / 2 \\
9 / 2 \\
11 / 2\end{array}$ & & $\begin{array}{l}88847 \\
89033 \\
89204 \\
89324\end{array}$ & & $\begin{array}{l}0.582 \\
0.990 \\
1.172 \\
1.267\end{array}$ \\
\hline$d^{5}$ & $c^{2} \mathrm{D}$ & $\begin{array}{l}5 / 2 \\
3 / 2\end{array}$ & & $\begin{array}{l}89509 \\
89557\end{array}$ & & $\begin{array}{l}1.200 \\
0.801\end{array}$ \\
\hline$d^{4} s$ & $\left(a^{3} \mathrm{P}\right)^{2} \mathrm{P}$ & $\begin{array}{l}1 / 2 \\
3 / 2\end{array}$ & & $\begin{array}{l}90229 \\
91310\end{array}$ & & $\begin{array}{l}0.675 \\
1.333\end{array}$ \\
\hline$d^{4} s$ & $\left({ }^{3} \mathrm{H}\right)^{2} \mathrm{H}$ & $\begin{array}{r}9 / 2 \\
11 / 2\end{array}$ & & $\begin{array}{l}90432 \\
90770\end{array}$ & & $\begin{array}{l}0.914 \\
1.095\end{array}$ \\
\hline$d^{4} s$ & $\left(a^{3} \mathrm{~F}\right)^{2} \mathrm{~F}$ & $\begin{array}{l}5 / 2 \\
7 / 2\end{array}$ & & $\begin{array}{l}91888 \\
91941\end{array}$ & & $\begin{array}{l}0.855 \\
1.132\end{array}$ \\
\hline$d^{4} s$ & $\left({ }^{3} \mathrm{G}\right)^{2} \mathrm{G}$ & $\begin{array}{l}7 / 2 \\
9 / 2\end{array}$ & & $\begin{array}{l}94395 \\
94722\end{array}$ & & $\begin{array}{l}0.895 \\
1.110\end{array}$ \\
\hline$d^{4} s$ & $\left({ }^{3} \mathrm{D}\right)^{4} \mathrm{D}$ & $\begin{array}{l}7 / 2 \\
5 / 2 \\
3 / 2 \\
1 / 2\end{array}$ & & $\begin{array}{l}94709 \\
94776 \\
94842 \\
94886\end{array}$ & & $\begin{array}{l}1.428 \\
1.371 \\
1.200 \\
0.003\end{array}$ \\
\hline
\end{tabular}


TABLE 8. Mn III-Observed and calculated energy levels - Continued

\begin{tabular}{|c|c|c|c|c|c|c|}
\hline CONF. & TERM & $J$ & OBS. & CALC. & $\mathrm{O}-\mathrm{C}$ & CALC. $g$ \\
\hline$d^{4} s$ & $\left(a^{1} \mathrm{G}\right)^{2} \mathrm{G}$ & $\begin{array}{l}9 / 2 \\
7 / 2\end{array}$ & & $\begin{array}{l}96358 \\
96400\end{array}$ & & $\begin{array}{l}1.111 \\
0.894\end{array}$ \\
\hline$d^{4} s$ & $\left({ }^{1} \mathrm{I}\right)^{2} \mathrm{I}$ & $\begin{array}{l}13 / 2 \\
11 / 2\end{array}$ & & $\begin{array}{l}97192 \\
97202\end{array}$ & & $\begin{array}{l}1.077 \\
0.924\end{array}$ \\
\hline$d^{4} s$ & $\left(a^{1} \mathbf{S}\right)^{2} \mathbf{S}$ & $1 / 2$ & & 98962 & & 1.996 \\
\hline$d^{4} s$ & $\left({ }^{3} \mathrm{D}\right)^{2} \mathrm{D}$ & $\begin{array}{l}5 / 2 \\
3 / 2\end{array}$ & & $\begin{array}{r}99976 \\
100057\end{array}$ & & $\begin{array}{l}1.200 \\
0.801\end{array}$ \\
\hline$d^{4} s$ & $\left(a^{1} \mathrm{D}\right)^{2} \mathrm{D}$ & $\begin{array}{l}5 / 2 \\
3 / 2\end{array}$ & & $\begin{array}{l}104407 \\
104436\end{array}$ & & $\begin{array}{l}1.200 \\
0.801\end{array}$ \\
\hline$d^{4} \mathrm{~s}$ & $\left({ }^{1} \mathrm{~F}\right)^{2} \mathrm{~F}$ & $\begin{array}{l}7 / 2 \\
5 / 2\end{array}$ & & $\begin{array}{l}109792 \\
109783\end{array}$ & & $\begin{array}{l}1.143 \\
0.858\end{array}$ \\
\hline$d^{4} s$ & $\left(b^{3} \mathrm{P}\right)^{4} \mathrm{P}$ & $\begin{array}{l}5 / 2 \\
3 / 2 \\
1 / 2\end{array}$ & & $\begin{array}{l}115528 \\
116093 \\
116452\end{array}$ & & $\begin{array}{l}1.599 \\
1.732 \\
2.664\end{array}$ \\
\hline$d^{4} s$ & $\left(b^{3} \mathrm{~F}\right)^{4} \mathrm{~F}$ & $\begin{array}{l}9 / 2 \\
7 / 2 \\
5 / 2 \\
3 / 2\end{array}$ & & $\begin{array}{l}115650 \\
115718 \\
115734 \\
115725\end{array}$ & & $\begin{array}{l}1.333 \\
1.237 \\
1.028 \\
0.401\end{array}$ \\
\hline$d^{4} s$ & $\left(b^{3} \mathrm{P}\right)^{2} \mathrm{P}$ & $\begin{array}{l}3 / 2 \\
1 / 2\end{array}$ & & $\begin{array}{l}120834 \\
121523\end{array}$ & & $\begin{array}{l}1.334 \\
0.669\end{array}$ \\
\hline$d^{4} s$ & $\left(b^{3} \mathrm{~F}\right)^{2} \mathrm{~F}$ & $\begin{array}{l}7 / 2 \\
5 / 2\end{array}$ & & $\begin{array}{l}120919 \\
120993\end{array}$ & & $\begin{array}{l}1.142 \\
0.857\end{array}$ \\
\hline$d^{4} s$ & $\left(b^{1} \mathrm{G}\right)^{2} \mathrm{G}$ & $\begin{array}{l}9 / 2 \\
7 / 2\end{array}$ & & $\begin{array}{l}124967 \\
124980\end{array}$ & & $\begin{array}{l}1.111 \\
0.890\end{array}$ \\
\hline$d^{4} s$ & $\left(b^{1} \mathrm{D}\right)^{2} \mathrm{D}$ & $\begin{array}{l}5 / 2 \\
3 / 2\end{array}$ & & $\begin{array}{l}143467 \\
143469\end{array}$ & & $\begin{array}{l}1.200 \\
0.800\end{array}$ \\
\hline$d^{4} s$ & $\left(b^{1} \mathbf{S}\right)^{2} \mathbf{S}$ & $1 / 2$ & & 165639 & & 2.000 \\
\hline
\end{tabular}

TABLE 9. Fe III-Observed and calculated energy levels

\begin{tabular}{|c|c|c|c|c|c|c|}
\hline CONF. & TERM & $J$ & OBS. & CALC. & $\mathrm{O}-\mathrm{C}$ & CALC. $g$ \\
\hline$d^{6}$ & ${ }^{5} \mathrm{D}$ & $\begin{array}{l}4 \\
3 \\
2 \\
1 \\
0\end{array}$ & $\begin{array}{r}0.0 \\
436.2 \\
738.9 \\
932.4 \\
1027.3\end{array}$ & $\begin{array}{r}-13 \\
419 \\
723 \\
918 \\
1014\end{array}$ & $\begin{array}{l}13 \\
17 \\
16 \\
14 \\
13\end{array}$ & $\begin{array}{l}1.500 \\
1.500 \\
1.500 \\
1.500 \\
0.000\end{array}$ \\
\hline$d^{6}$ & $a^{3} \mathrm{P}$ & $\begin{array}{l}2 \\
1 \\
0\end{array}$ & $\begin{array}{l}19404.8 \\
20688.4 \\
21208.5\end{array}$ & $\begin{array}{l}19427 \\
20703 \\
21238\end{array}$ & $\begin{array}{l}-22 \\
-15 \\
-30\end{array}$ & $\begin{array}{l}1.499 \\
1.499 \\
0.000\end{array}$ \\
\hline$d^{6}$ & ${ }^{3} \mathrm{H}$ & $\begin{array}{l}6 \\
5 \\
4\end{array}$ & $\begin{array}{l}20051.1 \\
20300.8 \\
20481.9\end{array}$ & $\begin{array}{l}20090 \\
20307 \\
20464\end{array}$ & $\begin{array}{r}-39 \\
-6 \\
18\end{array}$ & $\begin{array}{l}1.166 \\
1.036 \\
0.810\end{array}$ \\
\hline$d^{6}$ & $a^{3} \mathrm{~F}$ & $\begin{array}{l}4 \\
3 \\
2\end{array}$ & $\begin{array}{l}21462.2 \\
21699.9 \\
21857.2\end{array}$ & $\begin{array}{l}21481 \\
21708 \\
21867\end{array}$ & $\begin{array}{r}-19 \\
-8 \\
-10\end{array}$ & $\begin{array}{l}1.237 \\
1.077 \\
0.668\end{array}$ \\
\hline$d^{6}$ & ${ }^{3} \mathrm{G}$ & $\begin{array}{l}5 \\
4 \\
3\end{array}$ & $\begin{array}{l}24558.8 \\
24940.9 \\
25142.4\end{array}$ & $\begin{array}{l}24566 \\
24944 \\
25126\end{array}$ & $\begin{array}{r}-7 \\
-3 \\
16\end{array}$ & $\begin{array}{l}1.198 \\
1.053 \\
0.757\end{array}$ \\
\hline$d^{5} s$ & $\left({ }^{6} \mathrm{~S}\right)^{7} \mathrm{~S}$ & 3 & 30088.84 & 29997 & 92 & 2.000 \\
\hline
\end{tabular}


TABLE 9. Fe III-Observed and calculated energy levels - Continued

\begin{tabular}{|c|c|c|c|c|c|c|}
\hline CONF. & TERM & $J$ & OBS. & CALC. & $\mathrm{O}-\mathrm{C}$ & CALC. $g$ \\
\hline$d^{6}$ & ${ }^{1} \mathrm{I}$ & 6 & 30356.2 & 30336 & 20 & 1.000 \\
\hline \multirow[t]{3}{*}{$d^{6}$} & ${ }^{3} \mathrm{D}$ & 1 & 30725.8 & 30690 & 36 & 0.501 \\
\hline & & 2 & 30716.2 & 30699 & 17 & 1.165 \\
\hline & & 3 & 30857.8 & 30837 & 21 & 1.333 \\
\hline$d^{6}$ & $a^{1} \mathrm{G}$ & 4 & 30886.4 & 30838 & 48 & 1.001 \\
\hline$d^{6}$ & $a^{1} \mathrm{~S}$ & 0 & 34812.4 & 34715 & 97 & 0.000 \\
\hline$d^{6}$ & $a^{1} \mathrm{D}$ & 2 & 35803.7 & 35787 & 17 & 1.002 \\
\hline$d^{5} s$ & $\left({ }^{6} \mathrm{~S}\right)^{5} \mathrm{~S}$ & 2 & 40999.87 & 40866 & 134 & 2.000 \\
\hline$d^{6}$ & ${ }^{1} \mathrm{~F}$ & 3 & 42896.9 & 42801 & 96 & 1.001 \\
\hline \multirow[t]{3}{*}{$d^{6}$} & $b^{3} \mathrm{P}$ & 0 & 49148 & 49168 & -20 & 0.000 \\
\hline & & 1 & 49576.9 & 49574 & 3 & 1.500 \\
\hline & & 2 & 50412.3 & 50410 & 2 & 1.499 \\
\hline \multirow[t]{3}{*}{$d^{6}$} & $b^{3} \mathrm{~F}$ & 2 & 50184.9 & 50197 & -12 & 0.667 \\
\hline & & 3 & 50295.2 & 50281 & 14 & 1.083 \\
\hline & & 4 & 50276.1 & 50242 & 34 & 1.249 \\
\hline$d^{6}$ & $b^{1} \mathrm{G}$ & 4 & 57221.7 & 57121 & 101 & 1.001 \\
\hline \multirow[t]{5}{*}{$d^{5} s$} & $\left({ }^{4} \mathrm{G}\right)^{5} \mathrm{G}$ & 6 & 63425.17 & 63504 & -79 & 1.333 \\
\hline & & 5 & 63466.39 & 63510 & -44 & 1.267 \\
\hline & & 4 & 63486.78 & 63501 & -14 & 1.150 \\
\hline & & 3 & 63494.00 & 63485 & 9 & 0.917 \\
\hline & & 2 & 63494.56 & 63468 & 27 & 0.334 \\
\hline \multirow[t]{3}{*}{$d^{5} s$} & $\left({ }^{4} \mathrm{P}\right)^{5} \mathrm{P}$ & 3 & 66464.64 & 66518 & -53 & 1.658 \\
\hline & & 2 & 66522.95 & 66570 & -47 & 1.819 \\
\hline & & 1 & 66591.68 & 66642 & -50 & 2.482 \\
\hline \multirow[t]{5}{*}{$d^{5} s$} & $\left({ }^{4} \mathrm{D}\right)^{5} \mathrm{D}$ & 4 & 69695.73 & 69746 & -50 & 1.499 \\
\hline & & 3 & 69836.83 & 69885 & -48 & 1.506 \\
\hline & & 2 & 69837.76 & 69872 & -34 & 1.512 \\
\hline & & 1 & 69788.19 & 69808 & -20 & 1.517 \\
\hline & & 0 & 69747.40 & 69757 & -10 & 0.000 \\
\hline \multirow[t]{3}{*}{$d^{5} s$} & $\left({ }^{4} \mathrm{G}\right)^{3} \mathrm{G}$ & 5 & 70694.03 & 70748 & -54 & 1.200 \\
\hline & & 4 & 70728.75 & 70747 & -18 & 1.051 \\
\hline & & & 70725.01 & 70713 & 12 & 0.751 \\
\hline \multirow[t]{3}{*}{$d^{5} s$} & $\left({ }^{4} \mathrm{P}\right)^{3} \mathrm{P}$ & 2 & 73727.64 & 73757 & -29 & 1.482 \\
\hline & & 1 & 73849.10 & 73879 & -30 & 1.471 \\
\hline & & 0 & 73935.96 & 73975 & -39 & 0.000 \\
\hline$d^{6}$ & $b^{1} \mathrm{D}$ & 2 & & 76714 & & 1.000 \\
\hline \multirow[t]{3}{*}{$d^{5} s$} & $\left({ }^{4} \mathrm{D}\right)^{3} \mathrm{D}$ & 3 & 76956.79 & 76994 & -37 & 1.332 \\
\hline & & 2 & 77102.43 & 77136 & -34 & 1.180 \\
\hline & & 1 & 77075.30 & 77096 & -21 & 0.526 \\
\hline \multirow[t]{3}{*}{$d^{5} s$} & $\left({ }^{2} \mathrm{I}\right)^{3} \mathrm{I}$ & 7 & 79840.12 & 79885 & -45 & 1.143 \\
\hline & & 6 & 79844.74 & 79842 & 3 & 1.025 \\
\hline & & 5 & 79860.42 & 79818 & 42 & 0.835 \\
\hline \multirow[t]{3}{*}{$d^{5} s$} & $76 \%\left(a^{2} \mathrm{D}\right)^{3} \mathrm{D}+16 \%\left(a^{2} \mathrm{~F}\right)^{3} \mathrm{~F}$ & 3 & 82382.87 & 82362 & 21 & 1.285 \\
\hline & $69 \%\left(a^{2} \mathrm{D}\right)^{3} \mathrm{D}+17 \%\left({ }^{4} \mathrm{~F}\right)^{5} \mathrm{~F}$ & 2 & 82410.94 & 82399 & 12 & 1.077 \\
\hline & $66 \%\left(a^{2} \mathrm{D}\right)^{3} \mathrm{D}+34 \%\left({ }^{4} \mathrm{~F}\right)^{5} \mathrm{~F}$ & 1 & 82494.88 & 82502 & -7 & 0.332 \\
\hline \multirow[t]{5}{*}{$d^{5} s$} & $\left({ }^{4} \mathrm{~F}\right)^{5} \mathrm{~F}$ & 5 & 83138.23 & 83208 & -70 & 1.398 \\
\hline & $\left({ }^{4} \mathrm{~F}\right)^{5} \mathrm{~F}$ & 4 & 83161.48 & 83222 & -61 & 1.347 \\
\hline & $\left({ }^{4} \mathrm{~F}\right)^{5} \mathrm{~F}$ & 3 & 83237.86 & 83289 & -51 & 1.241 \\
\hline & $77 \%\left({ }^{4} \mathrm{~F}\right)^{5} \mathrm{~F}+15 \%\left(a^{2} \mathrm{~F}\right)^{3} \mathrm{~F}$ & 2 & 83358.88 & 83395 & -36 & 0.964 \\
\hline & $66 \%\left({ }^{4} \mathrm{~F}\right)^{5} \mathrm{~F}+34 \%\left(a^{2} \mathrm{D}\right)^{3} \mathrm{D}$ & 1 & 83646.98 & 83680 & -33 & 0.172 \\
\hline$d^{5} s$ & $\left({ }^{2} \mathrm{I}\right)^{1} \mathrm{I}$ & 6 & 83429.61 & $|83450|$ & -20 & 1.002 \\
\hline
\end{tabular}


TABLE 9. Fe III-Observed and calculated energy levels-Continued

\begin{tabular}{|c|c|c|c|c|c|c|}
\hline CONF. & TERM & $J$ & OBS. & CALC. & $\mathrm{O}-\mathrm{C}$ & CALC. $g$ \\
\hline \multirow[t]{3}{*}{$d^{5} s$} & $\left(a^{2} \mathrm{~F}\right)^{3} \mathrm{~F}$ & 4 & 84159.55 & 84162 & -3 & 1.251 \\
\hline & $77 \%\left(a^{2} \mathrm{~F}\right)^{3} \mathrm{~F}+18 \%\left(a^{2} \mathrm{D}\right)^{3} \mathrm{D}$ & 3 & 84671.87 & 84656 & 16 & 1.131 \\
\hline & $60 \%\left(a^{2} \mathrm{~F}\right)^{3} \mathrm{~F}+17 \%\left(a^{2} \mathrm{D}\right)^{3} \mathrm{D}$ & 2 & 84369.92 & 84347 & 23 & 0.834 \\
\hline $\mathrm{d}^{5} s$ & $\left(a^{2} \mathrm{D}\right)^{1} \mathrm{D}$ & 2 & 86847.11 & 86856 & -9 & 0.958 \\
\hline$d^{5} s$ & $\left(a^{2} \mathrm{~F}\right)^{1} \mathrm{~F}$ & 3 & 87901.87 & 87889 & 13 & 1.003 \\
\hline \multirow[t]{3}{*}{$d^{5} \mathrm{~s}$} & $\left({ }^{2} \mathrm{H}\right)^{3} \mathrm{H}$ & 4 & 88663.87 & 88612 & 52 & 0.835 \\
\hline & & 5 & 88694.67 & 88666 & 29 & 1.058 \\
\hline & & 6 & 88923.07 & 88921 & 2 & 1.164 \\
\hline \multirow[t]{3}{*}{$d^{5} \mathrm{~s}$} & $\left(a^{2} \mathrm{G}\right)^{3} \mathrm{G}$ & 3 & 89697.52 & 89698 & -1 & 0.783 \\
\hline & & 4 & 89783.59 & 89786 & -3 & 1.044 \\
\hline & & 5 & 89907.85 & 89906 & 2 & 1.172 \\
\hline \multirow[t]{3}{*}{$d^{5} s$} & $\left({ }^{4} \mathrm{~F}\right)^{3} \mathrm{~F}$ & 2 & 90423.68 & 90499 & -75 & 0.673 \\
\hline & & 3 & 90483.94 & 90551 & -67 & 1.058 \\
\hline & & 4 & 90472.53 & 90534 & -62 & 1.216 \\
\hline$d^{5} s$ & $\left({ }^{2} \mathrm{H}\right)^{1} \mathrm{H}$ & 5 & 92523.91 & 92526 & -2 & 1.004 \\
\hline \multirow[t]{3}{*}{$d^{5} s$} & $58 \%\left(b^{2} \mathrm{~F}\right)^{3} \mathrm{~F}+41 \%\left(a^{2} \mathrm{G}\right)^{1} \mathrm{G}$ & 4 & 93388.75 & 93409 & -20 & 1.193 \\
\hline & $\left(b^{2} \mathrm{~F}\right)^{3} \mathrm{~F}$ & 3 & 93392.45 & 93381 & 11 & 1.084 \\
\hline & $\left(b^{2} \mathrm{~F}\right)^{3} \mathrm{~F}$ & 2 & 93412.93 & 93387 & 26 & 0.668 \\
\hline$d^{5} s$ & $55 \%\left(a^{2} \mathrm{G}\right)^{1} \mathrm{G}+40 \%\left(b^{2} \mathrm{~F}\right)^{3} \mathrm{~F}$ & 4 & 93512.64 & 93498 & 15 & 1.064 \\
\hline$d^{5} s$ & $\left(b^{2} \mathrm{~F}\right)^{1} \mathrm{~F}$ & 3 & 97041.38 & 97038 & 3 & 1.001 \\
\hline$d^{6}$ & $b^{1} \mathrm{~S}$ & 0 & & 98216 & & 0.000 \\
\hline$d^{5} s$ & $\left({ }^{2} \mathrm{~S}\right)^{3} \mathrm{~S}$ & 1 & & 98666 & & 2.000 \\
\hline$d^{5} s$ & $\left({ }^{2} \mathrm{~S}\right)^{1} \mathrm{~S}$ & 0 & & 102289 & & 0.000 \\
\hline \multirow[t]{3}{*}{$d^{5} s$} & $\left(b^{2} \mathrm{D}\right)^{3} \mathrm{D}$ & 1 & 105895.35 & 105829 & 66 & 0.500 \\
\hline & & 2 & 105906.23 & 105864 & 42 & 1.166 \\
\hline & & 3 & 105929.16 & 105924 & 5 & 1.332 \\
\hline $\mathrm{d}^{5} s$ & $\left(b^{2} \mathrm{D}\right)^{1} \mathrm{D}$ & 2 & 109570.84 & 109507 & 64 & 1.000 \\
\hline \multirow[t]{3}{*}{$d^{5} s$} & $\left(b^{2} \mathrm{G}\right)^{3} \mathrm{G}$ & 5 & 114325.35 & 114284 & 41 & 1.200 \\
\hline & & 4 & 114339.95 & 114307 & 33 & 1.050 \\
\hline & & 3 & 114351.92 & 114325 & 27 & 0.750 \\
\hline$d^{5} s$ & $\left(b^{2} \mathrm{G}\right)^{1} \mathrm{G}$ & 4 & 117950.32 & 117922 & 28 & 1.000 \\
\hline \multirow[t]{3}{*}{$d^{5} s$} & $\left({ }^{2} \mathrm{P}\right)^{3} \mathrm{P}$ & 2 & & 130711 & & 1.499 \\
\hline & & 1 & & 130741 & & 1.499 \\
\hline & & 0 & & 130761 & & 0.000 \\
\hline$d^{5} s$ & $\left({ }^{2} \mathrm{P}\right)^{1} \mathrm{P}$ & 1 & & 134330 & & 0.996 \\
\hline \multirow[t]{3}{*}{$d^{5} s$} & $\left(c^{2} \mathrm{D}\right)^{3} \mathrm{D}$ & 3 & & 138594 & & 1.333 \\
\hline & & 2 & & 138644 & & 1.167 \\
\hline & & 1 & & 138694 & & 0.506 \\
\hline$d^{5} s$ & $\left(c^{2} \mathrm{D}\right)^{1} \mathrm{D}$ & 2 & & 142241 & & 1.000 \\
\hline
\end{tabular}

TABLE 10. Co III-Observed and calculated energy levels

\begin{tabular}{|c|c|c|c|c|c|c|}
\hline CONF. & TERM & $J$ & OBS. & CALC. & $\mathrm{O}-\mathrm{C}$ & CALC. $g$ \\
\hline$d^{7}$ & ${ }^{4} \mathrm{~F}$ & $\begin{array}{l}9 / 2 \\
7 / 2 \\
5 / 2 \\
3 / 2\end{array}$ & $\begin{array}{r}0.0 \\
841.2 \\
1451.3 \\
1866.8\end{array}$ & $\begin{array}{r}-17 \\
819 \\
1432 \\
1851\end{array}$ & $\begin{array}{l}17 \\
22 \\
19 \\
16\end{array}$ & $\begin{array}{l}1.333 \\
1.238 \\
1.029 \\
0.401\end{array}$ \\
\hline
\end{tabular}


TABLE 10. Co III-Observed and calculated energy levels-Continued

\begin{tabular}{|c|c|c|c|c|c|c|}
\hline CONF. & TERM & $J$ & OBS. & CALC. & O-C & CALC. $g$ \\
\hline$d^{7}$ & ${ }^{4} \mathrm{P}$ & $\begin{array}{l}5 / 2 \\
3 / 2 \\
1 / 2\end{array}$ & $\begin{array}{l}15201.9 \\
15428.2 \\
15811.4\end{array}$ & $\begin{array}{l}15200 \\
15447 \\
15861\end{array}$ & $\begin{array}{r}2 \\
-19 \\
-50\end{array}$ & $\begin{array}{l}1.599 \\
1.715 \\
2.631\end{array}$ \\
\hline$d^{7}$ & ${ }^{2} \mathrm{G}$ & $\begin{array}{l}9 / 2 \\
7 / 2\end{array}$ & $\begin{array}{l}16977.6 \\
17766.2\end{array}$ & $\begin{array}{l}16950 \\
17751\end{array}$ & $\begin{array}{l}28 \\
15\end{array}$ & $\begin{array}{l}1.108 \\
0.889\end{array}$ \\
\hline$d^{7}$ & ${ }^{2} \mathrm{P}$ & $\begin{array}{l}3 / 2 \\
1 / 2\end{array}$ & $\begin{array}{l}20194.9 \\
20918.5\end{array}$ & $\begin{array}{l}20196 \\
20931\end{array}$ & $\begin{array}{r}-1 \\
-13\end{array}$ & $\begin{array}{l}1.306 \\
0.702\end{array}$ \\
\hline$d^{7}$ & ${ }^{2} \mathrm{H}$ & $\begin{array}{r}11 / 2 \\
9 / 2\end{array}$ & $\begin{array}{l}22720.3 \\
23434.3\end{array}$ & $\begin{array}{l}22703 \\
23415\end{array}$ & $\begin{array}{l}17 \\
19\end{array}$ & $\begin{array}{l}1.091 \\
0.913\end{array}$ \\
\hline$d^{7}$ & $a^{2} \mathrm{D}$ & $\begin{array}{l}5 / 2 \\
3 / 2\end{array}$ & $\begin{array}{l}23058.8 \\
24236.8\end{array}$ & $\begin{array}{l}23032 \\
24217\end{array}$ & $\begin{array}{l}27 \\
20\end{array}$ & $\begin{array}{l}1.201 \\
0.845\end{array}$ \\
\hline $\begin{array}{l}d^{7} \\
d^{6} s\end{array}$ & $\begin{array}{l}{ }^{2} \mathrm{~F} \\
\left({ }^{5} \mathrm{D}\right)^{6} \mathrm{D}\end{array}$ & $\begin{array}{l}5 / 2 \\
7 / 2 \\
9 / 2 \\
7 / 2 \\
5 / 2 \\
3 / 2 \\
1 / 2\end{array}$ & $\begin{array}{l}37021.0 \\
37316.5 \\
46438.3 \\
47003.1 \\
47415.4 \\
47698.6 \\
47864.8\end{array}$ & $\begin{array}{l}36946 \\
37302 \\
46444 \\
46997 \\
47404 \\
47686 \\
47851\end{array}$ & $\begin{array}{r}75 \\
15 \\
-6 \\
6 \\
11 \\
13 \\
14\end{array}$ & $\begin{array}{l}0.858 \\
1.143 \\
1.555 \\
1.587 \\
1.657 \\
1.866 \\
3.331\end{array}$ \\
\hline$d^{6} s$ & $\left({ }^{5} \mathrm{D}\right)^{4} \mathrm{D}$ & $\begin{array}{l}7 / 2 \\
5 / 2 \\
3 / 2 \\
1 / 2\end{array}$ & $\begin{array}{l}55729.2 \\
56373.8 \\
56794.8 \\
57036.8\end{array}$ & $\begin{array}{l}55705 \\
56347 \\
56770 \\
57014\end{array}$ & $\begin{array}{l}24 \\
27 \\
25 \\
23\end{array}$ & $\begin{array}{l}1.428 \\
1.371 \\
1.200 \\
0.003\end{array}$ \\
\hline$d^{7}$ & $b^{2} \mathrm{D}$ & $\begin{array}{l}3 / 2 \\
1 / 2\end{array}$ & & $\begin{array}{l}57142 \\
57567\end{array}$ & & $\begin{array}{l}0.800 \\
1.200\end{array}$ \\
\hline$d^{6} s$ & $\left(a^{3} \mathrm{P}\right)^{4} \mathrm{P}$ & $\begin{array}{l}5 / 2 \\
3 / 2 \\
1 / 2\end{array}$ & $\begin{array}{l}70934.1 \\
72341.9 \\
73214.5\end{array}$ & $\begin{array}{l}70933 \\
72337 \\
73225\end{array}$ & $\begin{array}{r}1 \\
5 \\
-11\end{array}$ & $\begin{array}{l}1.599 \\
1.722 \\
2.653\end{array}$ \\
\hline$d^{6} \mathrm{~s}$ & $\left({ }^{3} \mathrm{H}\right)^{4} \mathrm{H}$ & $\begin{array}{r}13 / 2 \\
11 / 2 \\
9 / 2 \\
7 / 2\end{array}$ & $\begin{array}{l}71623.1 \\
71873.7 \\
72083.3 \\
72270.5\end{array}$ & $\begin{array}{l}71683 \\
71890 \\
72063 \\
72222\end{array}$ & $\begin{array}{r}-60 \\
-16 \\
20 \\
49\end{array}$ & $\begin{array}{l}1.230 \\
1.135 \\
0.978 \\
0.676\end{array}$ \\
\hline$d^{6} \mathrm{~s}$ & $\left(a^{3} \mathrm{~F}\right)^{4} \mathrm{~F}$ & $\begin{array}{l}9 / 2 \\
7 / 2 \\
5 / 2 \\
3 / 2\end{array}$ & $\begin{array}{l}73286.0 \\
73540.2 \\
73726.6 \\
73861.8\end{array}$ & $\begin{array}{l}73311 \\
73546 \\
73724 \\
73856\end{array}$ & $\begin{array}{r}-25 \\
-6 \\
3 \\
6\end{array}$ & $\begin{array}{l}1.322 \\
1.225 \\
1.021 \\
0.402\end{array}$ \\
\hline$d^{6} s$ & $\left({ }^{3} \mathrm{G}\right)^{4} \mathrm{G}$ & $\begin{array}{r}11 / 2 \\
9 / 2 \\
7 / 2 \\
5 / 2\end{array}$ & $\begin{array}{l}76518.9 \\
77121.1 \\
77383.1 \\
77472.3\end{array}$ & $\begin{array}{l}76564 \\
77153 \\
77405 \\
77470\end{array}$ & $\begin{array}{r}-45 \\
-32 \\
-22 \\
2\end{array}$ & $\begin{array}{l}1.222 \\
1.133 \\
0.994 \\
0.596\end{array}$ \\
\hline$d^{6} \mathrm{~s}$ & $\left(a^{3} \mathrm{P}\right)^{2} \mathrm{P}$ & $\begin{array}{l}3 / 2 \\
1 / 2\end{array}$ & $\begin{array}{l}76791.1 \\
78434.3\end{array}$ & $\begin{array}{l}76786 \\
78444\end{array}$ & $\begin{array}{r}5 \\
-10\end{array}$ & $\begin{array}{l}1.341 \\
0.679\end{array}$ \\
\hline$d^{6} \mathrm{~s}$ & $\left({ }^{3} \mathrm{H}\right)^{2} \mathrm{H}$ & $\begin{array}{r}11 / 2 \\
9 / 2\end{array}$ & $\begin{array}{l}77411.6 \\
77622.9\end{array}$ & $\begin{array}{l}77464 \\
77624\end{array}$ & $\begin{array}{r}-52 \\
-1\end{array}$ & $\begin{array}{l}1.138 \\
0.955\end{array}$ \\
\hline$d^{6} s$ & $\left(a^{3} \mathbf{F}\right)^{2} \mathrm{~F}$ & $\begin{array}{l}7 / 2 \\
5 / 2\end{array}$ & $\begin{array}{l}78927.8 \\
79425.3\end{array}$ & $\begin{array}{l}78940 \\
79436\end{array}$ & $\begin{array}{l}-12 \\
-11\end{array}$ & $\begin{array}{l}1.126 \\
0.843\end{array}$ \\
\hline$d^{6} s$ & $\left({ }^{3} \mathrm{G}\right)^{2} \mathrm{G}$ & $\begin{array}{l}9 / 2 \\
7 / 2\end{array}$ & $\begin{array}{l}82363.3 \\
82920.7\end{array}$ & $\begin{array}{l}82409 \\
82947\end{array}$ & $\begin{array}{l}-46 \\
-26\end{array}$ & $\begin{array}{l}1.108 \\
0.897\end{array}$ \\
\hline$d^{6} s$ & $\left({ }^{3} \mathrm{D}\right)^{4} \mathrm{D}$ & $\begin{array}{l}1 / 2 \\
3 / 2 \\
5 / 2 \\
7 / 2\end{array}$ & $\begin{array}{l}83789.3 \\
83773.4 \\
83799.6 \\
83938.9\end{array}$ & $\begin{array}{l}83796 \\
83795 \\
83837 \\
83982\end{array}$ & $\begin{array}{r}-7 \\
-22 \\
-37 \\
-43\end{array}$ & $\begin{array}{l}0.006 \\
1.199 \\
1.370 \\
1.428\end{array}$ \\
\hline$d^{6} \mathrm{~s}$ & $\left({ }^{1} \mathrm{I}\right)^{2} \mathrm{I}$ & $\begin{array}{l}13 / 2 \\
11 / 2\end{array}$ & $\begin{array}{l}85474.1 \\
85517.3\end{array}$ & $\begin{array}{l}85468 \\
85486\end{array}$ & $\begin{array}{r}6 \\
31\end{array}$ & $\begin{array}{l}1.077 \\
0.924\end{array}$ \\
\hline
\end{tabular}


TABLE 10. Co III-Observed and calculated energy levels-Continued

\begin{tabular}{|c|c|c|c|c|c|c|}
\hline CONF. & TERM & $J$ & OBS. & CALC. & $\mathrm{O}-\mathrm{C}$ & CALC. $g$ \\
\hline$d^{6} s$ & $\left(a^{1} \mathrm{G}\right)^{2} \mathrm{G}$ & $\begin{array}{l}9 / 2 \\
7 / 2\end{array}$ & $\begin{array}{l}86283.8 \\
86327.1\end{array}$ & $\begin{array}{l}86291 \\
86317\end{array}$ & $\begin{array}{r}-7 \\
10\end{array}$ & $\begin{array}{l}1.111 \\
0.892\end{array}$ \\
\hline$d^{6} s$ & $\left({ }^{3} \mathrm{D}\right)^{2} \mathrm{D}$ & $\begin{array}{l}3 / 2 \\
5 / 2\end{array}$ & & $\begin{array}{l}89277 \\
89535\end{array}$ & & $\begin{array}{l}0.802 \\
1.199\end{array}$ \\
\hline$d^{6} s$ & $\left(a^{1} \mathrm{~S}\right)^{2} \mathrm{~S}$ & $1 / 2$ & & 90884 & & 1.996 \\
\hline$d^{6} s$ & $\left(a^{1} \mathrm{D}\right)^{2} \mathrm{D}$ & $\begin{array}{l}5 / 2 \\
3 / 2\end{array}$ & 91715.1 & $\begin{array}{l}91673 \\
91780\end{array}$ & 42 & $\begin{array}{l}1.201 \\
0.802\end{array}$ \\
\hline$d^{6} s$ & $\left({ }^{1} \mathrm{~F}\right)^{2} \mathrm{~F}$ & $\begin{array}{l}7 / 2 \\
5 / 2\end{array}$ & & $\begin{array}{l}99597 \\
99588\end{array}$ & & $\begin{array}{l}1.144 \\
0.859\end{array}$ \\
\hline$d^{6} s$ & $\left(b^{3} \mathrm{P}\right)^{4} \mathrm{P}$ & $\begin{array}{l}1 / 2 \\
3 / 2 \\
5 / 2\end{array}$ & & $\begin{array}{l}105113 \\
105671 \\
106698\end{array}$ & & $\begin{array}{l}2.661 \\
1.731 \\
1.598\end{array}$ \\
\hline$d^{6} s$ & $\left(b^{3} \mathrm{~F}\right)^{4} \mathrm{~F}$ & $\begin{array}{l}3 / 2 \\
5 / 2 \\
7 / 2 \\
9 / 2\end{array}$ & & $\begin{array}{l}106482 \\
106565 \\
106607 \\
106528\end{array}$ & & $\begin{array}{l}0.401 \\
1.029 \\
1.237 \\
1.333\end{array}$ \\
\hline$d^{6} s$ & $\left(b^{3} \mathrm{P}\right)^{2} \mathrm{P}$ & $\begin{array}{l}1 / 2 \\
3 / 2\end{array}$ & & $\begin{array}{l}111013 \\
112214\end{array}$ & & $\begin{array}{l}0.672 \\
1.334\end{array}$ \\
\hline$d^{6} s$ & $\left(b^{3} \mathrm{~F}\right)^{2} \mathrm{~F}$ & $\begin{array}{l}7 / 2 \\
5 / 2\end{array}$ & & $\begin{array}{l}112119 \\
112144\end{array}$ & & $\begin{array}{l}1.140 \\
0.858\end{array}$ \\
\hline$d^{6} s$ & $\left(b^{1} G\right)^{2} G$ & $\begin{array}{l}9 / 2 \\
7 / 2\end{array}$ & & $\begin{array}{l}116336 \\
116365\end{array}$ & & $\begin{array}{l}1.112 \\
0.892\end{array}$ \\
\hline$d^{6} s$ & $\left(b^{1} \mathrm{D}\right)^{2} \mathrm{D}$ & $\begin{array}{l}3 / 2 \\
5 / 2\end{array}$ & & $\begin{array}{l}138788 \\
138785\end{array}$ & & $\begin{array}{l}0.800 \\
1.200\end{array}$ \\
\hline$d^{6} s$ & $\left(b^{1} \mathrm{~S}\right)^{2} \mathrm{~S}$ & $1 / 2$ & & 164007 & & 2.000 \\
\hline
\end{tabular}

TABLE 11. NIIII-Observed and calculated energy levels

\begin{tabular}{|c|c|c|c|c|c|c|}
\hline CONF. & TERM & $J$ & OBS. & C.ALC. & $\mathrm{O}-\mathrm{C}$ & C.ALC. $g$ \\
\hline$d^{8}$ & ${ }^{3} \mathrm{~F}$ & $\begin{array}{l}4 \\
3 \\
2\end{array}$ & $\begin{array}{r}0.0 \\
1360.7 \\
2269.8\end{array}$ & $\begin{array}{l}-12 \\
1361 \\
2284\end{array}$ & $\begin{array}{r}12 \\
-0 \\
-14\end{array}$ & $\begin{array}{l}1.250 \\
1.083 \\
0.669\end{array}$ \\
\hline$d^{8}$ & ${ }^{1} \mathrm{D}$ & 2 & 14031.6 & 14039 & -7 & 1.078 \\
\hline$d^{8}$ & ${ }^{3} \mathrm{P}$ & $\begin{array}{l}2 \\
1 \\
0\end{array}$ & $\begin{array}{l}16661.6 \\
16977.8 \\
17230.7\end{array}$ & $\begin{array}{l}16705 \\
16955 \\
17216\end{array}$ & $\begin{array}{r}-43 \\
23 \\
15\end{array}$ & $\begin{array}{l}1.420 \\
1.500 \\
0.000\end{array}$ \\
\hline$d^{8}$ & ${ }^{1}($ & 4 & 23108.7 & 23091 & 18 & 1.000 \\
\hline$d^{8}$ & is & 0 & & 52564 & & 0.000 \\
\hline$d^{7} s$ & $\left({ }^{4} \mathrm{~F}\right)^{5} \mathrm{~F}$ & $\begin{array}{l}5 \\
4 \\
3 \\
2 \\
1\end{array}$ & $\begin{array}{l}53703.7 \\
54657.9 \\
55406.1 \\
55952.0 \\
56308.2\end{array}$ & $\begin{array}{l}53722 \\
54659 \\
55399 \\
55941 \\
56296\end{array}$ & $\begin{array}{r}-18 \\
-1 \\
7 \\
11 \\
12\end{array}$ & $\begin{array}{l}1.399 \\
1.349 \\
1.249 \\
1.000 \\
0.002\end{array}$ \\
\hline$d^{7} s$ & $\left({ }^{4} \mathrm{~F}\right)^{3} \mathrm{~F}$ & $\begin{array}{l}4 \\
3 \\
2\end{array}$ & $\begin{array}{l}61339.2 \\
62606.3 \\
63472.6\end{array}$ & $\begin{array}{l}61338 \\
62602 \\
63472\end{array}$ & $\begin{array}{l}1 \\
4 \\
1\end{array}$ & $\begin{array}{l}1.249 \\
1.084 \\
0.669\end{array}$ \\
\hline$d^{7} s$ & $\left({ }^{4} P^{P}\right)^{5} P^{3}$ & $\begin{array}{l}3 \\
2 \\
1\end{array}$ & $\begin{array}{l}71067.0 \\
71383.7 \\
718+1.9\end{array}$ & $\begin{array}{r}71061 \\
71397 \\
971886\end{array}$ & $\begin{array}{r}6 \\
-13 \\
-4\end{array}$ & $\begin{array}{l}1.666 \\
1.822 \\
2.479\end{array}$ \\
\hline
\end{tabular}


TABLE 11. Observed and calculated levels-Continued

\begin{tabular}{|c|c|c|c|c|c|c|}
\hline CONF. & TERM & $J$ & OBS. & CALC. & $\mathrm{O}-\mathrm{C}$ & CALC. $g$ \\
\hline$d^{7} s$ & $\left({ }^{2} \mathrm{G}\right)^{3} \mathrm{G}$ & $\begin{array}{l}5 \\
4 \\
3\end{array}$ & $\begin{array}{l}75123.8 \\
75646.9 \\
76237.8\end{array}$ & $\begin{array}{l}75141 \\
75652 \\
76246\end{array}$ & $\begin{array}{r}-17 \\
-5 \\
-8\end{array}$ & $\begin{array}{l}1.197 \\
1.045 \\
0.751\end{array}$ \\
\hline$d^{7} s$ & $\begin{array}{l}47 \%\left({ }^{4} \mathrm{P}\right)^{3} \mathrm{P}+53 \%\left({ }^{2} \mathrm{P}\right)^{3} \mathrm{P} \\
52 \%\left({ }^{4} \mathrm{P}\right){ }^{3} \mathrm{P}+35 \%\left({ }^{2} \mathrm{P}\right){ }^{3} \mathrm{P} \\
48 \%\left({ }^{4} \mathrm{P}\right)^{3} \mathrm{P}+46 \%\left({ }^{2} \mathrm{P}\right)^{3} \mathrm{P}\end{array}$ & $\begin{array}{l}2 \\
1 \\
0\end{array}$ & $\begin{array}{l}78303.1 \\
78482.9 \\
78657.8\end{array}$ & $\begin{array}{l}78285 \\
78465 \\
78647\end{array}$ & $\begin{array}{l}18 \\
18 \\
11\end{array}$ & $\begin{array}{l}1.488 \\
1.441 \\
0.000\end{array}$ \\
\hline$d^{7} s$ & $\begin{array}{l}47 \%\left({ }^{2} \mathrm{P}\right)^{3} \mathrm{P}+53 \%\left({ }^{4} \mathrm{P}\right)^{3} \mathrm{P} \\
56 \%\left({ }^{2} \mathrm{P}\right)^{3} \mathrm{P}+39 \%\left({ }^{4} \mathrm{P}\right)^{3} \mathrm{P} \\
43 \%\left({ }^{2} \mathrm{P}\right)^{3} \mathrm{P}+51 \%\left({ }^{4} \mathrm{P}\right)^{3} \mathrm{P}\end{array}$ & $\begin{array}{l}2 \\
1 \\
0\end{array}$ & 79143.3 & $\begin{array}{l}79129 \\
79758 \\
80669\end{array}$ & 14 & $\begin{array}{l}1.492 \\
1.469 \\
0.000\end{array}$ \\
\hline$d^{7} s$ & $\left({ }^{2} \mathrm{G}\right)^{1} \mathrm{G}$ & 4 & 79250.8 & 79248 & 3 & 0.993 \\
\hline$d^{7} s$ & $\left({ }^{2} \mathrm{H}\right)^{3} \mathrm{H}$ & $\begin{array}{r}6 \\
5 \\
4\end{array}$ & $\begin{array}{l}81686.9 \\
82194.3 \\
82826.9\end{array}$ & $\begin{array}{l}81703 \\
82191 \\
82821\end{array}$ & $\begin{array}{c}-16 \\
3 \\
6\end{array}$ & $\begin{array}{l}1.167 \\
1.035 \\
0.814\end{array}$ \\
\hline$d^{7} s$ & $\begin{array}{l}\left(a^{2} \mathrm{D}\right)^{3} \mathrm{D} \\
\left(a^{2} \mathrm{D}\right)^{3} \mathrm{D} \\
54 \%\left(a^{2} \mathrm{D}\right)^{3} \mathrm{D}+41 \%\left({ }^{2} \mathrm{P}\right)^{1} \mathrm{P}\end{array}$ & $\begin{array}{l}3 \\
2 \\
1\end{array}$ & $\begin{array}{l}82172.9 \\
83033.8 \\
82277.0\end{array}$ & $\begin{array}{l}82183 \\
83038 \\
82273\end{array}$ & $\begin{array}{r}-10 \\
-4 \\
4\end{array}$ & $\begin{array}{l}1.334 \\
1.177 \\
0.852\end{array}$ \\
\hline$d^{7} s$ & $48 \%\left({ }^{2} \mathrm{P}\right)^{1} \mathrm{P}+40 \%\left(a^{2} \mathrm{D}\right)^{1} \mathrm{D}$ & 1 & 84604.8 & 84.628 & -23 & 0.758 \\
\hline$d^{7} s$ & $\left({ }^{2} \mathrm{H}\right)^{1} \mathrm{H}$ & 5 & 85834.8 & 85838 & -3 & 1.002 \\
\hline$d^{7} s$ & $\left(a^{2} \mathrm{D}\right)^{1} \mathrm{D}$ & 2 & 86646.2 & 86646 & 0 & 1.019 \\
\hline$d^{7} s$ & $\left({ }^{2} \mathrm{~F}\right)^{3} \mathrm{~F}$ & $\begin{array}{l}2 \\
3 \\
4\end{array}$ & $\begin{array}{l}97841.4 \\
97996.3 \\
98238.2\end{array}$ & $\begin{array}{l}97796 \\
97989 \\
98294\end{array}$ & $\begin{array}{r}45 \\
7 \\
-56\end{array}$ & $\begin{array}{l}0.668 \\
1.083 \\
1.250\end{array}$ \\
\hline$d^{7} s$ & $\left({ }^{2} \mathrm{~F}\right)^{1} \mathrm{~F}$ & 3 & & 101989 & & 1.001 \\
\hline$d^{7} s$ & $\left(b^{2} \mathrm{D}\right)^{3} \mathrm{D}$ & $\begin{array}{l}1 \\
2 \\
3\end{array}$ & $\begin{array}{l}121412.2 \\
121802.8\end{array}$ & $\begin{array}{l}121189 \\
121401 \\
121770\end{array}$ & $\begin{array}{l}11 \\
33\end{array}$ & $\begin{array}{l}0.500 \\
1.166 \\
1.333\end{array}$ \\
\hline$d^{7} s$ & $\left(b^{2} \mathrm{D}\right)^{1} \mathrm{D}$ & 2 & & 125444 & & 1.001 \\
\hline
\end{tabular}

TABLE 12. CuIII-Observed and calculated energy levels

\begin{tabular}{|c|c|c|c|c|c|c|}
\hline CONF. & TERM & $J$ & OBS. & CALC. & $\mathrm{O}-\mathrm{C}$ & CALC. $g$ \\
\hline$d^{9}$ & ${ }^{2} \mathrm{D}$ & $\begin{array}{l}5 / 2 \\
3 / 2\end{array}$ & $\begin{array}{r}0.0 \\
2071.8\end{array}$ & $\begin{array}{r}30 \\
2111\end{array}$ & $\begin{array}{l}-30 \\
-39\end{array}$ & $\begin{array}{l}1.200 \\
0.800\end{array}$ \\
\hline$d^{8} s$ & $\left({ }^{3} \mathrm{~F}\right)^{4} \mathrm{~F}$ & $\begin{array}{l}9 / 2 \\
7 / 2 \\
5 / 2 \\
3 / 2\end{array}$ & $\begin{array}{l}60803.68 \\
62063.64 \\
63142.16 \\
63884.97\end{array}$ & $\begin{array}{l}60785 \\
62036 \\
63114 \\
63860\end{array}$ & $\begin{array}{l}19 \\
28 \\
28 \\
25\end{array}$ & $\begin{array}{l}1.333 \\
1.235 \\
1.027 \\
0.403\end{array}$ \\
\hline$d^{8} s$ & $\left({ }^{3} \mathrm{~F}\right)^{2} \mathrm{~F}$ & $\begin{array}{l}7 / 2 \\
5 / 2\end{array}$ & $\begin{array}{l}67015.29 \\
68962.23\end{array}$ & $\begin{array}{l}66992 \\
68951\end{array}$ & $\begin{array}{l}23 \\
11\end{array}$ & $\begin{array}{l}1.145 \\
0.863\end{array}$ \\
\hline$d^{8} s$ & $\begin{array}{l}50 \%\left({ }^{1} \mathrm{D}\right)^{2} \mathrm{D}+50 \%\left({ }^{3} \mathrm{P}\right)^{4} \mathrm{P} \\
78 \%\left({ }^{1} \mathrm{D}\right)^{2} \mathrm{D}+18 \%\left({ }^{3} \mathrm{P}\right)^{4} \mathrm{P}\end{array}$ & $\begin{array}{l}5 / 2 \\
3 / 2\end{array}$ & $\begin{array}{l}77966.37 \\
78778.22\end{array}$ & $\begin{array}{l}77979 \\
78761\end{array}$ & $\begin{array}{r}-13 \\
17\end{array}$ & $\begin{array}{l}1.402 \\
0.989\end{array}$ \\
\hline$d^{8} s$ & $\begin{array}{l}\left({ }^{3} \mathrm{P}\right)^{4} \mathrm{P} \\
82 \%\left({ }^{3} \mathrm{P}\right)^{4} \mathrm{P}+17 \%\left({ }^{1} \mathrm{D}\right)^{2} \mathrm{D} \\
50 \%\left({ }^{3} \mathrm{P}\right)^{4} \mathrm{P}+49 \%\left({ }^{1} \mathrm{D}\right)^{2} \mathrm{D}\end{array}$ & $\begin{array}{l}1 / 2 \\
3 / 2 \\
5 / 2\end{array}$ & $\begin{array}{l}80421.57 \\
80303.83 \\
80550.29\end{array}$ & $\begin{array}{l}80386 \\
80283 \\
80589\end{array}$ & $\begin{array}{r}36 \\
21 \\
-39\end{array}$ & $\begin{array}{l}2.664 \\
1.558 \\
1.393\end{array}$ \\
\hline$d^{8} s$ & $\left({ }^{3} \mathrm{P}\right)^{2} \mathrm{P}^{\mathrm{P}}$ & $\begin{array}{l}3 / 2 \\
1 / 2\end{array}$ & $\begin{array}{l}85445.42 \\
86131.99\end{array}$ & $\begin{array}{l}85515 \\
86142\end{array}$ & $\begin{array}{l}-70 \\
-10\end{array}$ & $\begin{array}{l}1.316 \\
0.670\end{array}$ \\
\hline$d^{\mathrm{x}} s$ & $\left({ }^{1} \mathrm{C}\right)^{2} \mathrm{G}$ & $\begin{array}{l}9 / 2 \\
7 / 2\end{array}$ & $\begin{array}{l}89016.57 \\
89044.87\end{array}$ & $\begin{array}{l}89032 \\
89037\end{array}$ & $\begin{array}{r}-16 \\
8\end{array}$ & $\begin{array}{l}1.111 \\
0.889\end{array}$ \\
\hline$d^{k} s$ & $\left({ }^{1} \mathrm{~S}\right)^{2} \mathrm{~S}$ & $1 / 2$ & & 122865 & & 2.000 \\
\hline
\end{tabular}


TABLE 13. Zn III-Observed and calculated energy levels

\begin{tabular}{l|ll|r|r|r|r|r}
\hline \hline \multicolumn{2}{c|}{ CONF. } & TERM & $J$ & \multicolumn{1}{c|}{ OBS. } & CALC. & O-C & CALC. $g$ \\
\hline$d^{10}$ & ${ }^{10} \mathrm{~S}$ & & 0 & 0 & 88 & -88 & 0.000 \\
$d^{9} s$ & $\left({ }^{2} \mathrm{D}\right)^{3} \mathrm{D}$ & & 3 & 78105 & 78092 & 13 & 1.333 \\
& & 2 & 79283 & 79255 & 28 & 1.148 \\
& & 1 & 80859 & 80817 & 42 & 0.500 \\
$d^{9} s$ & $\left({ }^{2} \mathrm{D}\right)^{1} \mathrm{D}$ & & 2 & 83509 & 83505 & 4 & 1.018 \\
\hline
\end{tabular}

\section{Discussion of the Results}

In L.S. 3 where the $\beta, T, T x$, and $P x$ corrections were included, we used 36 free parameters for fitting 334 observed levels to the calculated ones, and the rms error is $38 \mathrm{~cm}^{-1}$. In the calculation, analogous to reference [3], where these new corrections were not included, and the same experimental levels were fitted to the theoretical ones, the rms error is $184 \mathrm{~cm}^{-1}$. This drastic difference in the mean error is a definite proof of the importance of the new corrections. Comparing these results with the analogous ones of the second spectra of the iron group, we see that in the third spectra, the effect of the new corrections is much stronger.

Now let us examine in more detail the behavior of the various effective interaction parameters: The mean value of the parameter $\alpha$, which was already included in the previous work [3], is now halved, while its linear coefficient doesn't change considerably. This parameter still assumes equal values for the two configurations $d^{n}$ and $d^{n-1} s$. The parameter $\beta$ is constant for all the spectra, and also assumes the same value for both configurations. The parameter $T$ is constant for all spectra, but assumes a larger value for the configurations $d^{n-1} s$ than it does for the configurations of the type $d^{n}$. The parameter $T x$ is equal for both configurations, but changes linearly with the atomic number. The parameter $P x$, by its very definition, is defined only for the $d^{n-1} s$ configurations. This parameter is constant along the sequence. The average value of the parameter ( $;$ doesn't change considerably, but the addition of $P x$ greatly reduced its linear coefficient, so that now it is almost constant along the sequence, which is more analogous to the behavior of ( $;$ in the second spectra. The behavior of all of the other parameters is practically the same as in reference [3].

The values which we obtained in the present paper for the parameters $\alpha, \beta$ and $T$ are of the same order of magnitude as those obtained for them in the calculations given in references [20] and [21]. The above mentioned behavior of the parameters $\alpha, \beta$ and $T$ is consistent with our efforts to explain them as mainly representing the perturbations caused to a configuration of the type $3 s^{2} 3 p^{6} 3 d^{n}$ by configurations characterized by the same set of principle quantum numbers $[20,21]$.
We will now examine the relative importance of the various effective interaction parameters in improving our approximation. In table 2, the results of various least-squares calculations are reported. In all of them, the same experimental material is included, and the same restrictions as in L.S. 3 are imposed on all of the parameters used in any of these calculations. They differ from each other by the set of effective interaction parameters used in them.

In column 1 of table 2 , numbers are assigned to the various least-squares calculations. In the second column, we report which effective-interaction parameters were included in each calculation. In the third column, the total number of free parameters is given; and in the fourth column, we report the rms error. Row I is analogous to reference [3], except for the above mentioned changes in the experimental material. The results of calculations 2 and 3 show that the introduction of each of the parameters B or $T$ alone, doesn't reduce the $\mathrm{rms}$ error considerably. However, in calculation 4 , we see that the simultaneous introduction of $\beta$ and $T$ causes a drastic decrease of the rms error from 184 to $61 \mathrm{~cm}^{-1}$. The calculation reported in row 5 is identical with L.S. $2 c$, and we see that the addition of the second three-body parameter $T x$ causes an additional reduction of the $\mathrm{rms}$ error from 61 to $46 \mathrm{~cm}^{-1}$. In Row 6 , we see the effect of also using the parameter $P x$ which is a threebody effective-interáction between two $d$ electrons and one $s$ electron. The rms error is reduced to $38 \mathrm{~cm}^{-1}$. Remembering that this parameter can improve only the levels belonging to the $d^{n-1} s$ configurations, it follows that the actual average improvement achieved by the addition is considerably larger than $8 \mathrm{~cm}^{-1}$ (which is the difference between the rms errors of calculations 5 and 6). Calculation 7 was performed in order to compare the relative importance of the two parameters $T$ and $T x$, which represent three-body effective-interactions only between equivalent $d$ electrons. Calculation 7 is analogous to calculation 4 , except that the three-body parameter $T$ was replaced by the three-body parameter $T x$. Comparison of the rms errors definitely shows how much more important $T$ is than $T x$. It should be noted that the conclusions obtained in reference [1] are qualitatively equal to our analogous present conclusions. However, in the present paper, the final agreement achieved between 
theory and experiment is much better than the agreement achieved in the second spectra.

It is also interesting to compare the effectiveness of the above mentioned various approximations for each of the two configurations $d^{n}$ and $d^{n-1} s$. In table 3 , we describe the results of the least-squares calculations performed separately for the observed levels of the $d^{n}$ and $d^{n-1} s$ configurations. For each of them, four different least-squares calculations were performed. In the first one, only the parameter $\alpha$ was included. In the second one, the parameters $\alpha$, $\beta$, and $T$ were included; in the third one, the complete set of effective-interaction parameters between $d$ electrons, $\alpha, \beta, T$, and $T x$, was included. In the fourth calculation, the parameter $P x$ was also added. We see that in the first calculation which is analogous to that used in the previous paper [3], the calculated levels of the $d^{n-1} s$ configurations are much more accurately calculated than those of $d^{n}$. However, during the two following stages of improving our approximation, as reported in Rows 2 and 3 , the $d^{n}$ configurations are much more strongly effected than are the $d^{n-1} s$ configurations. Thus, the rms error is $35 \mathrm{~cm}^{-1}$ for all of the levels of the $d^{n}$ configurations, and $46 \mathrm{~cm}^{-1}$ for all of the levels of the $d^{n-1} \mathrm{~s}$ configurations. This result is very plausible since $\alpha, \beta$, $T$ and $T x$ constitute a complete set of effectiveinteractions between $3 d$ electrons only. In the calculation reported in Row 4, where the effective-interaction which includes an $s$ electron was also added, the $d^{n-1} s$ configurations were strongly improved, and are even somewhat better than the $d^{n}$ configurations.

We would like to note that after the addition of $P x$, we again tried to include in our calculations also the electrostatic interaction between the configurations $d^{n}$ and $d^{n-1} s$. This was done by the same procedures as those described in section 3. The effect of the improvement of the approximation was that, in the least-squares calculation, $H$ assumed more or less consistent values, with uncertainties somewhat smaller that the values themselves. These values of $H$ are quite similar to those obtained for $H$ in the second spectra of the iron group. Although this behaviour of $H$ is a remarkable improvement in comparison to its behaviour in the calculations described in section 3 of the present paper, its introduction into our calculation hardly reduced the $\mathrm{rms}$ error.

In these circumstances, we thought it unreasonable to add the parameter $P y$ and $P z$ (defined in section 4) which represent correction terms to the electrostatic configuration interaction. Thus, we have the impression that the process of improving the approximation by adding electrostatic effective-interaction correction terms has come to a standstill, at least at the present stage.

A Remark on the Second Spectra. We would like to note that we also tried to use the new parameter $P x$ in our calculations of the second spectra of the iron group. It didn't cause any significant improvement in the approximation; and naturally, also couldn't unambiguously determine the value of the parameter $P x$. This negative result was actually anticipated, since in reference [1], where $P x$ was not included, the $d^{n-1} s$ configurations are already more accurately approximated than are the $d^{n}$ configurations.

We thank Serge Feneuille for the information with which he kindly supplied us.

\section{References}

[1] Shadmi, Y., Oreg, J., and Stein, J., J. Opt. Soc. Am. 58, 909(1968).

[2] Racah, G., and Shadmi, Y., Bull. Res. Council Israel 8F, 15(1959).

[3] Shadmi, Y., Bull. Res. Council Israel 10F, 3(1962).

[4] Shadmi, Y., Bull. Res. Ciouncil Israel 9F, 4 (1961).

[5] Shadmi, Y., J. Res. NBS 70A (Phys. and Chem) No. 5, 435 (1966).

[6] Bacher, R. F., and Goudsmit, S. A., Phys. Rev. 46, 948 (1934).

[7] Trees, R. E., Phys. Rev. 83, 756 (1951).

[8] Trees, R. E., Phys. Rev. 84, 1089 (19.51).

[9] Trees, R. E., Phys. Rev. 129, 1220 (1963).

[10] Racah, (,., Phys. Rev. 85, 381 (1952).

[11] Racah, G., and Shadmi, Y., Phys. Rev. 119, 156 (1960).

[12] Rajnak, K., and Wybourne, B. G., Phys. Rev. 132, 280 (1963).

[13] Racah, (;., and Stein, J., Phys. Rev. 156, 58 (1967).

[14] Judd, B. R., Physica 33, 174 (1967).

[15] Feneuille, S., C. R. Acad. Sc. Paris 262, 23 (1966).

[16] Rarah, (;., Phys. Rev. 63, 367 (1943).

[17] Moore, C. E., Atomic Energy Levels, NBS Circ. No. 467, 1 and 2 (1949, 1952).

[18] Roth, C.., Ph. D. Thesis, The Hebrew University of Jerusalem, (1967).

[19] Shenstone, A. (;., Can. J. Phys. 38, 677 (1960).

[20] Shadmi, Y., Phys. Rev. 139, A43 (1965).

[21] Shadmi, Y., Physica 33, 183 (1967).

(Paper 73A2-542) 